\title{
Alpha effect and turbulent diffusion from convection
}

\author{
P. J. Käpylä ${ }^{1}$, M. J. Korpi ${ }^{1}$, and A. Brandenburg ${ }^{2}$ \\ 1 Observatory, Tähtitorninmäki, PO Box 14, 00014 University of Helsinki, Finland \\ e-mail: petri.kapyla@helsinki.fi \\ 2 NORDITA, AlbaNova University Center, Roslagstullsbacken 23, 10691 Stockholm, Sweden
}

Received 10 December 2008 / Accepted 18 March 2009

\begin{abstract}
Aims. We study turbulent transport coefficients that describe the evolution of large-scale magnetic fields in turbulent convection. Methods. We use the test field method, together with three-dimensional numerical simulations of turbulent convection with shear and rotation, to compute turbulent transport coefficients describing the evolution of large-scale magnetic fields in mean-field theory in the kinematic regime. We employ one-dimensional mean-field models with the derived turbulent transport coefficients to examine whether they give results that are compatible with direct simulations.

Results. The results for the $\alpha$-effect as a function of rotation rate are consistent with earlier numerical studies, i.e. increasing magnitude as rotation increases and approximately $\cos \theta$ latitude profile for moderate rotation. Turbulent diffusivity, $\eta_{\mathrm{t}}$, is proportional to the square of the turbulent vertical velocity in all cases. Whereas $\eta_{\mathrm{t}}$ decreases approximately inversely proportional to the wavenumber of the field, the $\alpha$-effect and turbulent pumping show a more complex behaviour with partial or full sign changes and the magnitude staying roughly constant. In the presence of shear and no rotation, a weak $\alpha$-effect is induced which does not seem to show any consistent trend as a function of shear rate. Provided that the shear is large enough, this small $\alpha$-effect is able to excite a dynamo in the mean-field model. The coefficient responsible for driving the shear-current effect shows several sign changes as a function of depth but is also able to contribute to dynamo action in the mean-field model. The growth rates in these cases are, however, well below those in direct simulations, suggesting that an incoherent $\alpha$-shear dynamo may also act in the simulations. If both rotation and shear are present, the $\alpha$-effect is more pronounced. At the same time, the combination of the shear-current and $\boldsymbol{\Omega} \times \boldsymbol{J}$-effects is also stronger than in the case of shear alone, but subdominant to the $\alpha$-shear dynamo. The results of direct simulations are consistent with mean-field models where all of these effects are taken into account without the need to invoke incoherent effects.
\end{abstract}

Key words. magnetohydrodynamics (MHD) - convection - turbulence - Sun: magnetic fields - stars: magnetic fields

\section{Introduction}

The solar magnetic field is thought to arise from a complicated interplay of turbulence, rotation, and large-scale shear flows (e.g. Ossendrijver 2003, and references therein). Whilst numerical simulations of simple systems using fully periodic boxes and externally forced idealised flows exhibiting large-scale dynamos have been around for some time (e.g. Brandenburg 2001, 2005a; Brandenburg et al. 2001; Mininni et al. 2005; Brandenburg \& Käpylä 2007; Yousef et al. 2008a,b; Käpylä \& Brandenburg 2009) and dynamos driven by the magnetorotational instability exhibit large-scale dynamos (e.g. Brandenburg et al. 1995; Hawley et al. 1996), convection simulations have not been able to produce appreciable large-scale magnetic fields until recently (Rotvig \& Jones 2002; Browning et al. 2006; Brown et al. 2007; Käpylä et al. 2008, hereafter Paper I; Hughes \& Proctor 2009). The main ingredient missing in many earlier simulations was a large-scale shear flow and boundary conditions which allow magnetic helicity fluxes out of the system. Indeed, the shear flow plays a dual role in dynamos: it not only generates new magnetic fields by stretching, but it also drives magnetic helicity fluxes along constant isocontours of shear which can allow efficient dynamo action (Vishniac \& Cho 2001; Brandenburg \& Subramanian 2005; Paper I). Recently, however, large-scale dynamos have also been found from rigidly rotating convection simulations without shear (Käpylä et al. 2009a).
Although large-scale magnetic fields can clearly be obtained from simulations, the origin of these fields in many cases (e.g. Yousef et al. 2008a,b; Paper I; Hughes \& Proctor 2009) is still uncertain. In the mean-field framework (e.g. Moffatt 1978; Parker 1979; Krause \& Rädler 1980; Rüdiger \& Hollerbach 2004), the dynamo process is described by turbulent transport coefficients that govern the evolution of large-scale magnetic field. The evolution equation for the large-scale part is obtained from the standard induction equation by decomposing magnetic and velocity fields into their mean and fluctuating parts, i.e. $\boldsymbol{B}=\overline{\boldsymbol{B}}+\boldsymbol{b}, \boldsymbol{U}=\overline{\boldsymbol{U}}+\boldsymbol{u}$, which leads to

$\frac{\partial \overline{\boldsymbol{B}}}{\partial t}=\nabla \times\left(\overline{\boldsymbol{U}} \times \overline{\boldsymbol{B}}+\overline{\boldsymbol{E}}-\eta \mu_{0} \overline{\boldsymbol{J}}\right)$

where $\eta$ is the molecular magnetic diffusivity, $\overline{\boldsymbol{J}}=\mu_{0}^{-1} \nabla \times \overline{\boldsymbol{B}}$ is the current density, and $\mu_{0}$ is the vacuum permeability. The remaining term, $\overline{\mathcal{E}} \equiv \overline{\boldsymbol{u} \times \boldsymbol{b}}$, is the electromotive force describing the effects of small-scale turbulence on the evolution of mean fields and can be represented in terms of the mean fields and their derivatives

$\overline{\mathcal{E}}_{i}=\alpha_{i j} \bar{B}_{j}+\eta_{i j k} \bar{B}_{j, k}+\ldots$,

where $\alpha_{i j}$ and $\eta_{i j k}$ are tensorial coefficients, commas denote partial derivatives, and summation over repeated indices is assumed. Expression (2) is valid if the mean fields vary slowly in space and time. 
Whilst mean-field models have been quite successful in reproducing many aspects of the solar magnetism (e.g. Ossendrijver 2003), they have often been hampered by the poor knowledge of the turbulent transport coefficients which could only be computed analytically using unrealistic or unjustified approximations, such as first order smoothing (FOSA). More recently, numerical models of convection in local Cartesian geometry have been employed to compute some of these coefficients in more realistic setups (Brandenburg et al. 1990; Ossendrijver et al. 2001, 2002; Giesecke et al. 2005; Käpylä et al. 2006a; Cattaneo \& Hughes 2006; Hughes \& Cattaneo 2008). To date, however, only coefficients relevant for the $\alpha_{i j}$ term in Eq. (2) have been determined from convection simulations. This is due to the limitations of the method used where a uniform magnetic field is imposed and the resulting electromotive force is measured. Furthermore, if the Lorentz force is retained in the simulations, dynamo-generated magnetic fields may grow to saturation, leading to quenching even if the imposed field is weak. At large magnetic Reynolds numbers such quenching can be very strong if there are no magnetic helicity fluxes, suggesting therefore small values of $\alpha$ even for weak imposed fields.

During recent years an improved scheme of extracting turbulent transport coefficients has appeared which is referred to as the test field method (Schrinner et al. 2005, 2007). In the test field method the velocity field of the simulation is used in a number of induction equations, which all correspond to a given set of large-scale test fields which do neither evolve nor react back onto the velocity field. The test fields are orthogonal so the coefficients can be obtained by matrix inversion. This method has been used successfully in setups where the turbulence is due to isotropic forcing without shear (Sur et al. 2008, Brandenburg et al. 2008b) and with shear (Brandenburg et al. 2008a; Mitra et al. 2009), respectively. Moreover, the method has been used to extract dynamo coefficients from more realistic setups where the turbulence is driven by supernovae (Gressel et al. 2008) and the magnetorotational instability (Brandenburg 2005b, 2008).

In the present paper we apply the method for the first time to convection simulations. We also seek to understand the dynamos reported in Paper I by applying the derived coefficients in a one-dimensional mean-field model. In the case of convection with rigid rotation it is likely that the large-scale fields are due to the turbulent $\alpha$-effect that is present in helical flows (Käpylä et al. 2009a). However, when shear is present, there are various mechanisms that can generate large-scale fields: in helical flows a finite $\alpha$-effect (e.g. Rädler et al. 2003; Rädler \& Stepanov 2006; Rüdiger \& Kitchatinov 2006) with shear can excite a classical $\alpha \Omega$ or $\alpha$-shear-dynamo (e.g. Brandenburg \& Käpylä 2007; Käpylä \& Brandenburg 2009). Even if the mean value of $\alpha$ is zero, strong enough fluctuations about zero in combination with shear can drive an incoherent $\alpha$-shear dynamo (e.g. Vishniac \& Brandenburg 1997; Proctor 2007). Finally, the shear-current (Rogachevskii \& Kleeorin 2003, 2004; Kleeorin \& Rogachevskii 2008) and $\boldsymbol{\Omega} \times \boldsymbol{J}$ (Rädler 1969; Rädler et al. 2003; Pipin 2008) effects may operate even in nonhelical turbulence. If both rotation and shear are present in the system it is not obvious how to distinguish between the shear-current and $\boldsymbol{\Omega} \times \boldsymbol{J}$ effects. In the present paper we are able to extract the relevant turbulent transport coefficients responsible for most of these processes and determine which one of them is dominant in the different cases with the help of a one-dimensional mean-field model. In order to facilitate comparisons between the mean-field models and the direct simulations presented in Paper I, we use identical setups and overlapping parameter regimes as those used in Paper I in the determination of the transport coefficients.

\section{Model and methods}

The setup is similar to that used by, e.g., Brandenburg et al. (1996), Ossendrijver et al. (2001, 2002), and Käpylä et al. (2004, 2006a) and in Paper I. A small rectangular portion of a star is modelled by a box situated at colatitude $\theta$. The coordinate system is such that $(x, y, z)$ corresponds to $(\theta, \phi, r)$ in a spherical coordinate system. The dimensions of the domain are in most cases $\left(L_{x}, L_{y}, L_{z}\right)=(4,4,2) d$, where $d$ is the depth of the convectively unstable layer, and it is also used as our unit length. The box is divided into three layers, an upper cooling layer, a convectively unstable layer, and a stable overshoot layer (see below). The following set of equations for compressible hydrodynamics is being solved:

$$
\begin{aligned}
& \frac{\mathcal{D} \ln \rho}{\mathcal{D} t}=-\boldsymbol{\nabla} \cdot \boldsymbol{U} \\
& \frac{\mathcal{D} \boldsymbol{U}}{\mathcal{D} t}=-S U_{x} \hat{\boldsymbol{y}}-\frac{1}{\rho} \boldsymbol{\nabla} p+\boldsymbol{g}-2 \mathbf{\Omega} \times \boldsymbol{U}+\frac{1}{\rho} \boldsymbol{\nabla} \cdot 2 v \rho \mathbf{S}, \\
& \frac{\mathcal{D} \boldsymbol{e}}{\mathcal{D} t}=-\frac{p}{\rho} \boldsymbol{\nabla} \cdot \boldsymbol{U}+\frac{1}{\rho} \boldsymbol{\nabla} \cdot K \boldsymbol{\nabla} T+2 \nu \mathbf{S}^{2}-\frac{e-e_{0}}{\tau(z)}
\end{aligned}
$$

where $\mathcal{D} / \mathcal{D} t=\partial / \partial t+\left(\boldsymbol{U}+\overline{\boldsymbol{U}}^{(S)}\right) \cdot \boldsymbol{\nabla}$, and $\overline{\boldsymbol{U}}^{(S)}=(0, S x, 0)$ is the imposed large-scale shear flow. The kinematic viscosity is given by $v, \rho$ is the density, $\boldsymbol{U}$ is the velocity, and $\boldsymbol{g}=-g \hat{z}$ is the gravitational acceleration. The fluid obeys an ideal gas law $p=\rho e(\gamma-1)$, where $p$ and $e$ are the pressure and internal energy, respectively, and $\gamma=c_{\mathrm{P}} / c_{\mathrm{V}}=5 / 3$ is the ratio of specific heats in constant pressure and volume. The internal energy is related to the temperature via $e=c_{\mathrm{V}} T$, and $K$ is the heat conductivity. The rate of strain tensor $\mathbf{S}$ is given by

$\mathrm{S}_{i j}=\frac{1}{2}\left(U_{j, i}+U_{i, j}\right)-\frac{1}{3} \delta_{i j} \boldsymbol{\nabla} \cdot \boldsymbol{U}$

The last term of Eq. (5) describes cooling at the top of the domain, where $\tau(z)$ is a cooling time which has a profile smoothly connecting the upper cooling layer and the convectively unstable layer below.

The coordinates $\left(z_{1}, z_{2}, z_{3}, z_{4}\right)=(-0.85,0,1,1.15) d$ give the vertical positions of the bottom of the box, the bottom and top of the convectively unstable layer, and the top of the box, respectively. We use a $K(z)$ profile such that the associated hydrostatic reference solution is piecewise polytropic with indices $\left(m_{1}, m_{2}, m_{3}\right)=(3,1,1)$. The cooling layer near the top makes that layer nearly isothermal and hence stably stratified. The bottom layer is also stably stratified, and the middle layer is convectively unstable.

Stress-free boundary conditions are used for the velocity,

$U_{x, z}=U_{y, z}=U_{z}=0$.

In the absence of shear the $x$ and $y$ directions are periodic whereas if shear is present, shearing-periodic conditions are used in the $x$ direction. A constant temperature gradient is maintained at the bottom of the box which leads to a steady influx of heat due to the constant heat conductivity. The simulations were made with the PENCIL CODE ${ }^{1}$, which uses sixth-order explicit finite differences in space and third order accurate time stepping method. Resolutions of up to $256^{3}$ mesh points were used.

\footnotetext{
1 http://www.nordita.org/software/pencil-code/
} 


\subsection{Units, nondimensional quantities, and parameters}

Dimensionless quantities are obtained by setting

$d=g=\rho_{0}=c_{\mathrm{P}}=\mu_{0}=1$,

where $\rho_{0}$ is the density at $z_{2}$. The units of length, time, velocity, density, entropy, and magnetic field are then

$$
\begin{aligned}
& {[x]=d, \quad[t]=\sqrt{d / g}, \quad[U]=\sqrt{d g}, \quad[\rho]=\rho_{0},} \\
& {[s]=c_{\mathrm{P}}, \quad[B]=\sqrt{d g \rho_{0} \mu_{0}} .}
\end{aligned}
$$

The simulations are then governed by the dimensionless numbers

$\operatorname{Pr}=\frac{v}{\chi_{0}}, \quad \operatorname{Re}=\frac{u_{\mathrm{rms}}}{v k_{\mathrm{f}}}, \quad \operatorname{Ra}=\frac{g d^{4}}{v \chi_{0}}\left(-\frac{1}{c_{\mathrm{P}}} \frac{\mathrm{d} s}{\mathrm{~d} z}\right)_{z_{\mathrm{m}}}$,

where $\chi_{0}=K /\left(\rho_{\mathrm{m}} c_{\mathrm{P}}\right)$ is the thermal diffusivity, $k_{\mathrm{f}}=2 \pi / d$ is an estimate of the wavenumber of the energy-carrying eddies, and $\rho_{\mathrm{m}}$ is the density in the middle of the unstable layer at $z_{\mathrm{m}}=\frac{1}{2}\left(z_{3}-z_{2}\right)$. Our choice of $k_{\mathrm{f}}$ is somewhat arbitrary because it is difficult to define a single length scale which would describe the flow in a highly inhomogeneous system such as stratified convection. The vertical extent of convective cells, however, is almost always of the order of the depth of the convectively unstable layer which suggests that $d$ could be used as the length scale describing convection. In the nonrotating case, this is also close to the horizontal size of the convective eddies. The entropy gradient, measured at $z_{\mathrm{m}}$ in the non-convecting initial state, is given by

$\left(-\frac{1}{c_{\mathrm{P}}} \frac{\mathrm{d} s}{\mathrm{~d} z}\right)_{z_{\mathrm{m}}}=\frac{\nabla-\nabla_{\mathrm{ad}}}{H_{\mathrm{P}}}$,

with $\nabla_{\mathrm{ad}}=1-1 / \gamma$ and $\nabla=(\partial \ln T / \partial \ln p)_{z_{\mathrm{m}}}$, and $H_{\mathrm{P}}$ is the pressure scale height.

The amount of stratification is determined by the parameter

$\xi_{0}=\frac{(\gamma-1) e_{0}}{g d}$

where $e_{0}$ is the internal energy at $z_{4}$. We use $\xi_{0}=1 / 3$ in all models.

\subsection{The test field method}

We employ the test field method (Schrinner et al. 2005, 2007), which is implemented into the PENCIL CODE, to determine turbulent transport coefficients. The uncurled induction equation in the shearing box approximation can be written in terms of the vector potential in the Weyl gauge as

$\frac{D A}{D t}=-S A_{y} \hat{\boldsymbol{x}}+\boldsymbol{U} \times \boldsymbol{B}-\eta \mu_{0} \boldsymbol{J}$,

where $D / D t=\partial / \partial t+S x \partial / \partial y, \boldsymbol{A}$ is the magnetic vector potential, and $\boldsymbol{B}=\boldsymbol{\nabla} \times \boldsymbol{A}$ is the magnetic field. The relative importance of magnetic diffusion over viscous and inertial forces can be characterized respectively in terms of magnetic Prandtl and Reynolds numbers

$\mathrm{Pm}=\frac{v}{\eta}, \quad \mathrm{Rm} \equiv \frac{u_{\mathrm{rms}}}{\eta k_{\mathrm{f}}}=\operatorname{Pm} \mathrm{Re}$.

In most cases we use $\mathrm{Pm}=5$ and $\mathrm{Rm} \approx 35$, see Table 1 . When we vary $\mathrm{Rm}$ in the range from roughly 1.5 to 150 , we keep
$\mathrm{Re} \approx 15$ and vary $\mathrm{Pm}$ in the range $0.1-10$. We decompose the fields into their mean and fluctuating parts according to

$\boldsymbol{A}=\overline{\boldsymbol{A}}+\boldsymbol{a}, \quad \boldsymbol{U}=\overline{\boldsymbol{U}}+\boldsymbol{u}, \quad \boldsymbol{B}=\overline{\boldsymbol{B}}+\boldsymbol{b}, \quad J=\bar{J}+j$,

where the overbars denote a horizontal average and lowercase quantities denote fluctuations around these averages. The equation for the mean vector potential is then

$\frac{D \overline{\boldsymbol{A}}}{D t}=-S \bar{A}_{y} \hat{\boldsymbol{x}}-\overline{\boldsymbol{U}} \times \overline{\boldsymbol{B}}+\overline{\boldsymbol{u} \times \boldsymbol{b}}-\eta \mu_{0} \overline{\boldsymbol{J}}$.

Subtracting (16) from (13) gives an equation for the fluctuating field which reads

$\frac{D \boldsymbol{a}}{D t}=-S a_{y} \hat{\boldsymbol{x}}+\overline{\boldsymbol{U}} \times \boldsymbol{b}+\boldsymbol{u} \times \overline{\boldsymbol{B}}+\boldsymbol{u} \times \boldsymbol{b}-\overline{\boldsymbol{u} \times \boldsymbol{b}}-\eta \mu_{0} \boldsymbol{j}$.

Instead of using the actual mean fields $\overline{\boldsymbol{B}}$ in this equations, they are replaced by orthogonal test fields $\overline{\boldsymbol{B}}^{p, q}$ and a separate Eq. (17) is solved for each one of them. Here we follow the same procedure as in Brandenburg et al. (2008a) and Mitra et al. (2009) and limit the study to mean magnetic fields that depend on $z$ only. We use test fields

$\overline{\boldsymbol{B}}^{1 c}=B_{0}(\cos k z, 0,0), \quad \overline{\boldsymbol{B}}^{2 c}=B_{0}(0, \cos k z, 0)$,

$\overline{\boldsymbol{B}}^{1 s}=B_{0}(\sin k z, 0,0), \quad \overline{\boldsymbol{B}}^{2 s}=B_{0}(0, \sin k z, 0)$,

where $k$ is the wavenumber of the test field. In most models we use $k / k_{1}=1$, where $k_{1}=2 \pi / L_{z}$. The electromotive force can be written as

$\overline{\mathcal{E}}_{i}=\alpha_{i j} \bar{B}_{j}-\eta_{i j} \mu_{0} \bar{J}_{j}$

where $\eta_{i 1}=\eta_{i 23}$ and $\eta_{i 2}=-\eta_{i 13}$. The $4+4$ coefficients are then obtained by inverting a simple matrix equation, relating the rank-2 tensor components to rank-3 tensor components.

Owing to the use of periodic boundary conditions in the horizontal directions, the $z$-component of the mean magnetic field is conserved and equal to the initial value, i.e. $\bar{B}_{3}=0$. Therefore the value of $\alpha_{33}$ is here of no interest.

It is convenient to discuss the results in terms of the quantities

$\gamma=\frac{1}{2}\left(\alpha_{21}-\alpha_{12}\right), \quad \epsilon_{\gamma}=\frac{1}{2}\left(\alpha_{21}+\alpha_{12}\right)$,

$\eta_{\mathrm{t}}=\frac{1}{2}\left(\eta_{11}+\eta_{22}\right), \epsilon_{\eta}=\frac{1}{2}\left(\eta_{11}-\eta_{22}\right)$,

$\delta=\frac{1}{2}\left(\eta_{21}-\eta_{12}\right)$.

Furthermore, the remaining or otherwise important coefficients are analyzed individually. The most important of these are the diagonal components of $\alpha_{i j}$ and $\eta_{21}$. The former are responsible for the generation of magnetic fields in helical turbulence and the latter can drive the mean-field shear-current dynamo in nonhelical turbulence with shear (Rogachevskii \& Kleeorin 2003, 2004).

To normalize our results, we use isotropic expressions of $\alpha$ and $\eta_{\mathrm{t}}$ as obtained from first order smoothing, i.e.

$\alpha_{0}=\frac{1}{3} u_{\mathrm{rms}}, \quad \eta_{\mathrm{t} 0}=\frac{1}{3} u_{\mathrm{rms}} k_{\mathrm{f}}^{-1}$,

where the root mean square velocity is a volume average and the Strouhal number,

$\mathrm{St}=\tau_{\mathrm{c}} u_{\mathrm{rms}} k_{\mathrm{f}}$,

has been assumed to be of the order of unity. In order to actually compare our results with those of FOSA, anisotropic expressions 
Table 1. Summary of the runs. The numbers are given for the statistically saturated state. Here, $\tilde{k}=k / k_{1}, \mathrm{Ma}=u_{\mathrm{rms}} /(g d)^{1 / 2}$, and $L_{\mathrm{H}}=L_{x}=L_{y}$.

\begin{tabular}{|c|c|c|c|c|c|c|c|c|c|c|c|}
\hline Run & grid & $\operatorname{Pr}$ & $\mathrm{Ra}$ & $\mathrm{Rm}$ & Pm & Sh & Co & $\theta$ & Ma & $\tilde{k}$ & $L_{\mathrm{H}}$ \\
\hline $\mathrm{A}$ & $128^{3}$ & 1.37 & $3.1 \times 10^{5}$ & 37 & 5 & 0 & 0 & - & 0.046 & 1 & 4 \\
\hline B & $128^{3}$ & 1.37 & $3.1 \times 10^{5}$ & 35 & 5 & 0 & 0.36 & $0^{\circ}$ & 0.043 & 1 & 4 \\
\hline $\mathrm{C}$ & $128^{3}$ & 1.37 & $3.1 \times 10^{5}$ & 46 & 5 & -0.14 & 0 & - & 0.058 & 1 & 4 \\
\hline D & $128^{3}$ & 1.37 & $3.1 \times 10^{5}$ & 35 & 5 & -0.18 & 0.36 & $0^{\circ}$ & 0.044 & 1 & 4 \\
\hline A1 & $128^{3}$ & 1.37 & $3.1 \times 10^{5}$ & 37 & 5 & 0 & 0 & - & 0.046 & 0 & 4 \\
\hline $\mathrm{A} 2$ & $128^{3}$ & 1.37 & $3.1 \times 10^{5}$ & 37 & 5 & 0 & 0 & - & 0.046 & 1 & 4 \\
\hline A3 & $128^{3}$ & 1.37 & $3.1 \times 10^{5}$ & 38 & 5 & 0 & 0 & - & 0.048 & 2 & 4 \\
\hline A4 & $128^{3}$ & 1.37 & $3.1 \times 10^{5}$ & 38 & 5 & 0 & 0 & - & 0.048 & 3 & 4 \\
\hline B1 & $64 \times 128^{2}$ & 1.37 & $3.1 \times 10^{5}$ & 33 & 5 & 0 & 0.38 & $0^{\circ}$ & 0.042 & 1 & 2 \\
\hline B2 & $128^{3}$ & 1.37 & $3.1 \times 10^{5}$ & 35 & 5 & 0 & 0.36 & $0^{\circ}$ & 0.043 & 1 & 4 \\
\hline B3 & $256 \times 128^{2}$ & 1.37 & $3.1 \times 10^{5}$ & 32 & 5 & 0 & 0.40 & $0^{\circ}$ & 0.040 & 1 & 8 \\
\hline B4 & $128^{3}$ & 0.69 & $6.1 \times 10^{5}$ & 1.6 & 0.1 & 0 & 0.32 & $0^{\circ}$ & 0.049 & 1 & 4 \\
\hline B5 & $128^{3}$ & 0.69 & $6.1 \times 10^{5}$ & 3.2 & 0.2 & 0 & 0.32 & $0^{\circ}$ & 0.050 & 1 & 4 \\
\hline B6 & $128^{3}$ & 0.69 & $6.1 \times 10^{5}$ & 7.8 & 0.5 & 0 & 0.33 & $0^{\circ}$ & 0.049 & 1 & 4 \\
\hline B7 & $128^{3}$ & 0.69 & $6.1 \times 10^{5}$ & 16 & 1 & 0 & 0.32 & $0^{\circ}$ & 0.050 & 1 & 4 \\
\hline B8 & $128^{3}$ & 0.69 & $6.1 \times 10^{5}$ & 32 & 2 & 0 & 0.32 & $0^{\circ}$ & 0.050 & 1 & 4 \\
\hline B9 & $128^{3}$ & 0.69 & $6.1 \times 10^{5}$ & 75 & 5 & 0 & 0.34 & $0^{\circ}$ & 0.047 & 1 & 4 \\
\hline B10 & $256^{3}$ & 0.69 & $6.1 \times 10^{5}$ & 155 & 10 & 0 & 0.33 & $0^{\circ}$ & 0.049 & 1 & 4 \\
\hline B11 & $128^{3}$ & 1.37 & $3.1 \times 10^{5}$ & 35 & 5 & 0 & 0.36 & $0^{\circ}$ & 0.044 & 0 & 4 \\
\hline B12 & $128^{3}$ & 1.37 & $3.1 \times 10^{5}$ & 35 & 5 & 0 & 0.36 & $0^{\circ}$ & 0.043 & 1 & 4 \\
\hline B13 & $128^{3}$ & 1.37 & $3.1 \times 10^{5}$ & 35 & 5 & 0 & 0.36 & $0^{\circ}$ & 0.045 & 2 & 4 \\
\hline B14 & $128^{3}$ & 1.37 & $3.1 \times 10^{5}$ & 35 & 5 & 0 & 0.36 & $0^{\circ}$ & 0.045 & 3 & 4 \\
\hline B15 & $128^{3}$ & 1.37 & $3.1 \times 10^{5}$ & 35 & 5 & 0 & 0.07 & $0^{\circ}$ & 0.044 & 1 & 4 \\
\hline B16 & $128^{3}$ & 1.37 & $3.1 \times 10^{5}$ & 33 & 5 & 0 & 0.15 & $0^{\circ}$ & 0.042 & 1 & 4 \\
\hline B17 & $128^{3}$ & 1.37 & $3.1 \times 10^{5}$ & 35 & 5 & 0 & 0.36 & $0^{\circ}$ & 0.044 & 1 & 4 \\
\hline B18 & $128^{3}$ & 1.37 & $3.1 \times 10^{5}$ & 33 & 5 & 0 & 0.78 & $0^{\circ}$ & 0.041 & 1 & 4 \\
\hline B19 & $128^{3}$ & 1.37 & $3.1 \times 10^{5}$ & 29 & 5 & 0 & 1.74 & $0^{\circ}$ & 0.037 & 1 & 4 \\
\hline B20 & $128^{3}$ & 1.37 & $3.1 \times 10^{5}$ & 20 & 5 & 0 & 6.43 & $0^{\circ}$ & 0.025 & 1 & 4 \\
\hline B21 & $128^{3}$ & 1.37 & $3.1 \times 10^{5}$ & 35 & 5 & 0 & 0.36 & $0^{\circ}$ & 0.044 & 1 & 4 \\
\hline B22 & $128^{3}$ & 1.37 & $3.1 \times 10^{5}$ & 36 & 5 & 0 & 0.35 & $15^{\circ}$ & 0.045 & 1 & 4 \\
\hline B23 & $128^{3}$ & 1.37 & $3.1 \times 10^{5}$ & 37 & 5 & 0 & 0.34 & $30^{\circ}$ & 0.046 & 1 & 4 \\
\hline B24 & $128^{3}$ & 1.37 & $3.1 \times 10^{5}$ & 38 & 5 & 0 & 0.33 & $45^{\circ}$ & 0.048 & 1 & 4 \\
\hline B25 & $128^{3}$ & 1.37 & $3.1 \times 10^{5}$ & 37 & 5 & 0 & 0.34 & $60^{\circ}$ & 0.047 & 1 & 4 \\
\hline B26 & $128^{3}$ & 1.37 & $3.1 \times 10^{5}$ & 40 & 5 & 0 & 0.32 & $75^{\circ}$ & 0.050 & 1 & 4 \\
\hline B27 & $128^{3}$ & 1.37 & $3.1 \times 10^{5}$ & 43 & 5 & 0 & 0.29 & $90^{\circ}$ & 0.054 & 1 & 4 \\
\hline $\mathrm{C} 1$ & $128^{3}$ & 1.37 & $3.1 \times 10^{5}$ & 43 & 5 & -0.03 & 0 & - & 0.054 & $\frac{1}{1}$ & 4 \\
\hline $\mathrm{C} 2$ & $128^{3}$ & 1.37 & $3.1 \times 10^{5}$ & 42 & 5 & -0.06 & 0 & - & 0.052 & 1 & 4 \\
\hline $\mathrm{C} 3$ & $128^{3}$ & 1.37 & $3.1 \times 10^{5}$ & 46 & 5 & -0.14 & 0 & - & 0.058 & 1 & 4 \\
\hline $\mathrm{C} 4$ & $128^{3}$ & 1.37 & $3.1 \times 10^{5}$ & 66 & 5 & -0.19 & 0 & - & 0.083 & 1 & 4 \\
\hline D1 & $128^{3}$ & 1.37 & $3.1 \times 10^{5}$ & 37 & 5 & -0.03 & 0.06 & $0^{\circ}$ & 0.046 & 1 & 4 \\
\hline D2 & $128^{3}$ & 1.37 & $3.1 \times 10^{5}$ & 37 & 5 & -0.07 & 0.15 & $0^{\circ}$ & 0.043 & 1 & 4 \\
\hline D3 & $128^{3}$ & 1.37 & $3.1 \times 10^{5}$ & 37 & 5 & -0.18 & 0.36 & $0^{\circ}$ & 0.044 & 1 & 4 \\
\hline D4 & $128^{3}$ & 1.37 & $3.1 \times 10^{5}$ & 37 & 5 & -0.36 & 0.73 & $0^{\circ}$ & 0.044 & 1 & 4 \\
\hline D5 & $128^{3}$ & 1.37 & $3.1 \times 10^{5}$ & 37 & 5 & -0.83 & 1.66 & $0^{\circ}$ & 0.038 & 1 & 4 \\
\hline
\end{tabular}

need to be used. Such expressions have been computed in the past (e.g. Rädler 1980; see also Käpylä et al. 2006a) and are given for the $\alpha$-effect, $\gamma$, and $\eta_{\mathrm{t}}$ by

$\alpha_{x x}^{(0)}=-2 \tau_{\mathrm{c}} \overline{u_{z} \partial_{x} u_{y}}$,

$\alpha_{y y}^{(0)}=-2 \tau_{\mathrm{c}} \overline{u_{x} \partial_{y} u_{z}}$,

$\gamma^{(0)}=-\tau_{\mathrm{c}} \partial_{z} \overline{u_{z}^{2}}$,

$\eta_{\mathrm{t} 0}^{(0)}=\tau_{\mathrm{c}} \overline{u_{z}^{2}}$,

where we have used integration by parts and assumed that $\tau_{\mathrm{c}}$ does not depend on spatial coordinates. The correlation time can be presented in terms $u_{\mathrm{rms}}$ and $k_{\mathrm{f}}$ by assuming a value for St.

\subsection{Averaging and error estimates}

In the present study a mean quantity is considered to be a horizontal average, defined via

$\overline{\boldsymbol{F}}=\frac{1}{L_{x} L_{y}} \int_{-\frac{1}{2} L_{y}}^{\frac{1}{2} L_{y}} \int_{-\frac{1}{2} L_{x}}^{\frac{1}{2} L_{x}} \boldsymbol{F}\left(x+x^{\prime}, y+y^{\prime}, z, t\right) \mathrm{d} x^{\prime} \mathrm{d} y^{\prime}$.

Except for special terms such as the shear terms in Eqs. (4) and (13), this formulation corresponds to simple horizontal averaging (for details see Brandenburg et al. 2008a). An additional time average over the statistically steady part of each simulations is also applied. The fluctuating magnetic fields $\boldsymbol{b}^{p, q}$ are reset to zero after periodic time intervals in order to avoid the complications arising from the growth of these fields; see the more thorough discussions in Sur et al. (2008) and Mitra et al. (2009). 
We estimate errors by computing the standard deviation $\sigma$ for each depth and dividing this by the square root of the number of independent realizations $N$ of the dynamo coefficients. We consider the time series between two resets of the field $\boldsymbol{b}^{p, q}$ to represent an independent realization. For a typical run, $N$ is between five and ten.

\subsection{Corresponding mean-field models}

In order to determine how well the derived dynamo coefficients describe the dynamos seen in direct simulations of Paper I, we construct a one-dimensional mean-field model where the test field results can be used directly as inputs. We start from the mean-field induction equation, Eq. (1), which can be written using the vector potential

$\dot{\bar{A}}_{i}=-\bar{U}_{j, i} \bar{A}_{j}+\alpha_{i j} \bar{B}_{j}-\left(\eta_{i j}+\eta \delta_{i j}\right) \mu_{0} \bar{J}_{j}$

where the dot on $\dot{\bar{A}}_{i}$ denotes a time derivative and $\bar{U}_{j, i} \bar{A}_{j}=$ $\left(S \bar{A}_{y}, 0,0\right)$ is the shear term. The mean magnetic field is given by $\overline{\boldsymbol{B}}=\left(-\bar{A}_{y}^{\prime}, \bar{A}_{x}^{\prime}, 0\right)$, the mean current density is given by $\mu_{0} \bar{J}_{i}=$ $-\bar{A}_{i}^{\prime \prime}$, and primes denote $z$-derivatives. The coefficients $\alpha_{i j}$ and $\eta_{i j}$ are taken directly from the test field results leaving little freedom in the model. We can, however, turn on and off any component of $\alpha_{i j}$ and $\eta_{i j}$ when needed in order to study the effects of the different coefficients individually.

\section{Results}

In a similar fashion as in Paper I we perform four types of simulations which we label as follows: in set A neither rotation nor shear is present whereas in set B rotation is added. In set C only shear is present, and finally in set D both rotation and shear are used. Parameters such as the strengths of rotation and shear, as measured by $\mathrm{Co}$ and $\mathrm{Sh}$, respectively, are varied within each set to probe the parameter space. Summary of the runs is presented in Table 1.

The fluid Reynolds numbers in our simulations are quite modest so we cannot consider our flows to be highly turbulent. However, the flows are irregular enough to remain time dependent in all cases, as can also be seen from various animations ${ }^{2}$.

\subsection{Set $A$ : no rotation nor shear $(\mathrm{Co}=\mathrm{Sh}=0)$}

The simplest case we can consider with the present setup is one with no rotation and no shear. In that case no net helicity generation or $\alpha$-effect are expected. However, due to the density stratification, the turbulence is inhomogeneous. This can lead to a non-zero pumping, or $\gamma$-effect (e.g. Krause \& Rädler 1980).

The horizontally and temporally averaged transport coefficients from Run A with $\mathrm{Co}=\mathrm{Sh}=0$ and $\mathrm{Rm} \approx 37$ are presented in Fig. 1. The results show that the kinetic helicity is small and that the mean values of the diagonal elements of $\alpha_{i j}$ are of the order of $0.1 \alpha_{0}$ with errors clearly larger than the mean. Vanishing diagonal elements of $\alpha_{i j}$ is in accordance with expectations from symmetry arguments. There is however a non-zero pumping effect directed upward (downward) in the lower (upper) part of the convectively unstable layer. The sign of the pumping is inconsistent with the diamagnetic effect, i.e. $\gamma \propto-\partial_{z} \overline{u_{z}^{2}}$ (e.g. Rädler 1968) and differs from earlier results from convection simulations using the imposed field method

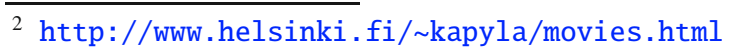
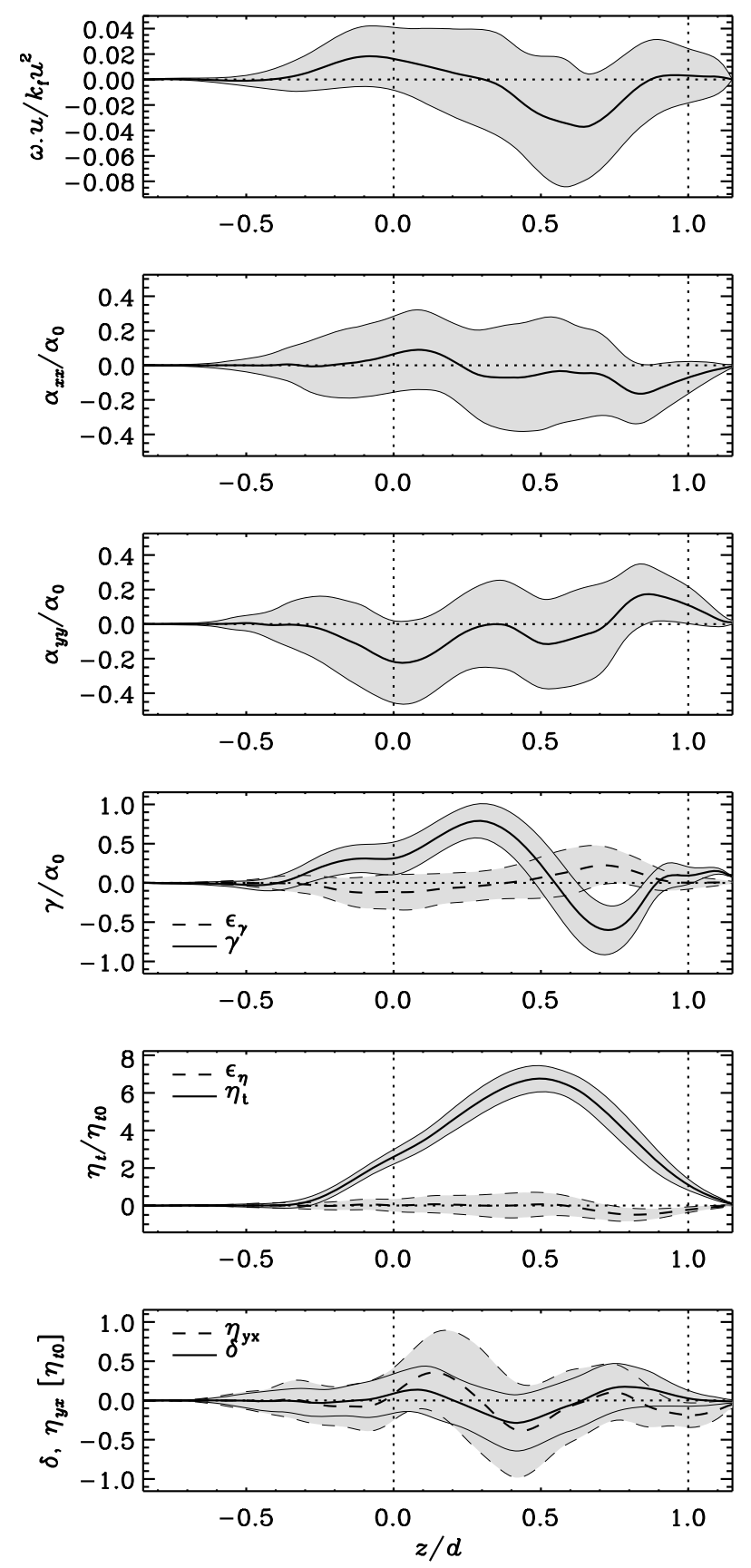

Fig. 1. The three topmost panels show the time-averaged vertical profiles of kinetic helicity, $\alpha_{x x}$, and $\alpha_{y y}$, respectively. The fourth and fifth panels show $\gamma$ with $\epsilon_{\gamma}$, and $\eta_{\mathrm{t}}$ with $\epsilon_{\eta}$, respectively. The lowermost panel shows $\delta$ (solid line) and $\eta_{y x}$ (dashed). From Run A with $\mathrm{Co}=\mathrm{Sh}=0$, and $\mathrm{Rm} \approx 37$. The shaded areas between the thinner lines indicate error estimates as described in Sect. 2.3. The vertical lines at $z=(0, d)$ denote the base and top of the convectively unstable layer.

(Ossendrijver et al. 2002; Käpylä et al. 2006a) and other diagnostics (e.g. Nordlund et al. 1992; Tobias et al. 1998, 2001; Ziegler \& Rüdiger 2003). However, this result is obtained for test fields for which $k / k_{1}=1$, whereas the imposed field results use a uniform field with $k / k_{1}=0$. For a uniform test field the pumping effect indeed changes sign and is thus consistent with the earlier numerical studies and the diamagnetic effect (see the upper panel of Fig. 2). The FOSA-prediction, Eq. (28) for the turbulent pumping is in qualitative agreement with the 

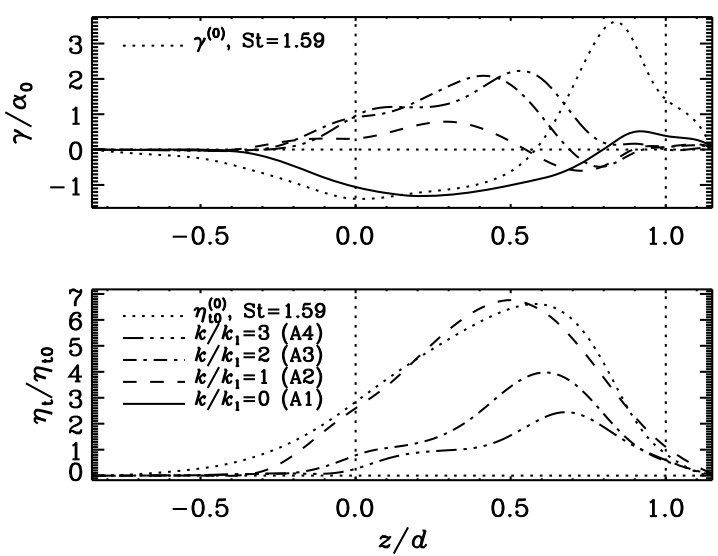

Fig. 2. Coefficients $\gamma$ (top panel) and $\eta_{\mathrm{t}}$ (bottom panel) as functions of $k$ from Runs $\mathrm{A} 1-\mathrm{A} 4$ with $\mathrm{Rm} \approx 37$, and $\mathrm{Co}=\mathrm{Sh}=0$. The dotted lines show the FOSA results for $\gamma$ and $\eta$, according to Eqs. (28) and (29), respectively, with $\mathrm{St} \approx 1.59$.

simulation result for $k / k_{1}=0$ but opposite to the results for $k / k_{1}$ greater than that.

At first glance the magnitude of the turbulent diffusivity seems quite high: the maximum value is more than six times the isotropic reference value $\eta_{\mathrm{t} 0}$ suggesting that $\mathrm{St} \approx 6$. However, the high value of $\eta_{\mathrm{t}}$ turns out to be related to the normalization: if an anisotropic expression, i.e. Eq. (29), is plotted alongside $\eta_{\mathrm{t}}$ the Strouhal number is roughly 1.6, not six, for our standard case $k / k_{1}=1$, see the lower panel of Fig. 2. The profile of the turbulent diffusivity coincides with that of the vertical velocity squared as predicted by Eq. (29). When $k$ is increased, the profile of $\eta_{\mathrm{t}}$ stays roughly the same and the magnitude diminishes roughly in proportion to $k^{-1}$. The quantities $\epsilon_{\gamma}, \epsilon_{\eta}, \delta, \eta_{x y}$, and $\eta_{y x}$ are compatible with zero in all runs in set $\mathrm{A}$.

\subsection{Set $B$ : only rotation $(C o \neq 0, S h=0)$}

When rotation (corresponding to the north pole, $\theta=0$ ) is added to the system, non-zero negative kinetic helicity is produced due to the fact that $\boldsymbol{g} \cdot \boldsymbol{\Omega}<0$. Although the $\alpha$-effect is not directly proportional to the helicity in the anisotropic case of stratified convection, it can still be a useful proxy. Figure 3 shows the results for Run $\mathrm{B}$ with $\mathrm{Co} \approx 0.36$ and $\mathrm{Rm} \approx 35$. We find that the diagonal components of $\alpha_{i j}$ are positive in the upper part of the convectively unstable region where the helicity is most negative. The negative maxima at the base of the convection zone are, however, not reflected by the kinetic helicity. The results in Fig. 3 were obtained for $k / k_{1}=1$. The profiles of $\alpha_{x x}$ and $\alpha_{y y}$ are more in line with the profile of the helicity for $k / k_{1}=0$ (see Sect. 3.2.3 for more details on the $k$-dependence).

In comparison to Run A, the pumping coefficient $\gamma$ shows a deeper maximum in the upper half of the convection zone and somewhat decreased value in the lower half. The profile and magnitude of the turbulent diffusivity are similar to those in the nonrotating case. The coefficients $\eta_{y x}$ and $\eta_{x y}$ are equal in magnitude and of opposite sign. This leads to a positive (negative) $\delta$ in the convection zone (overshoot layer) with magnitude peaking close to twice $\eta_{\mathrm{t} 0}$. The quantities $\epsilon_{\gamma}$ and $\epsilon_{\eta}$ are small, as expected from symmetry arguments

\subsubsection{Dependence on horizontal system size}

The profiles and magnitudes of the two diagonal components of $\alpha$ are very close to each other in our standard case (Run B)
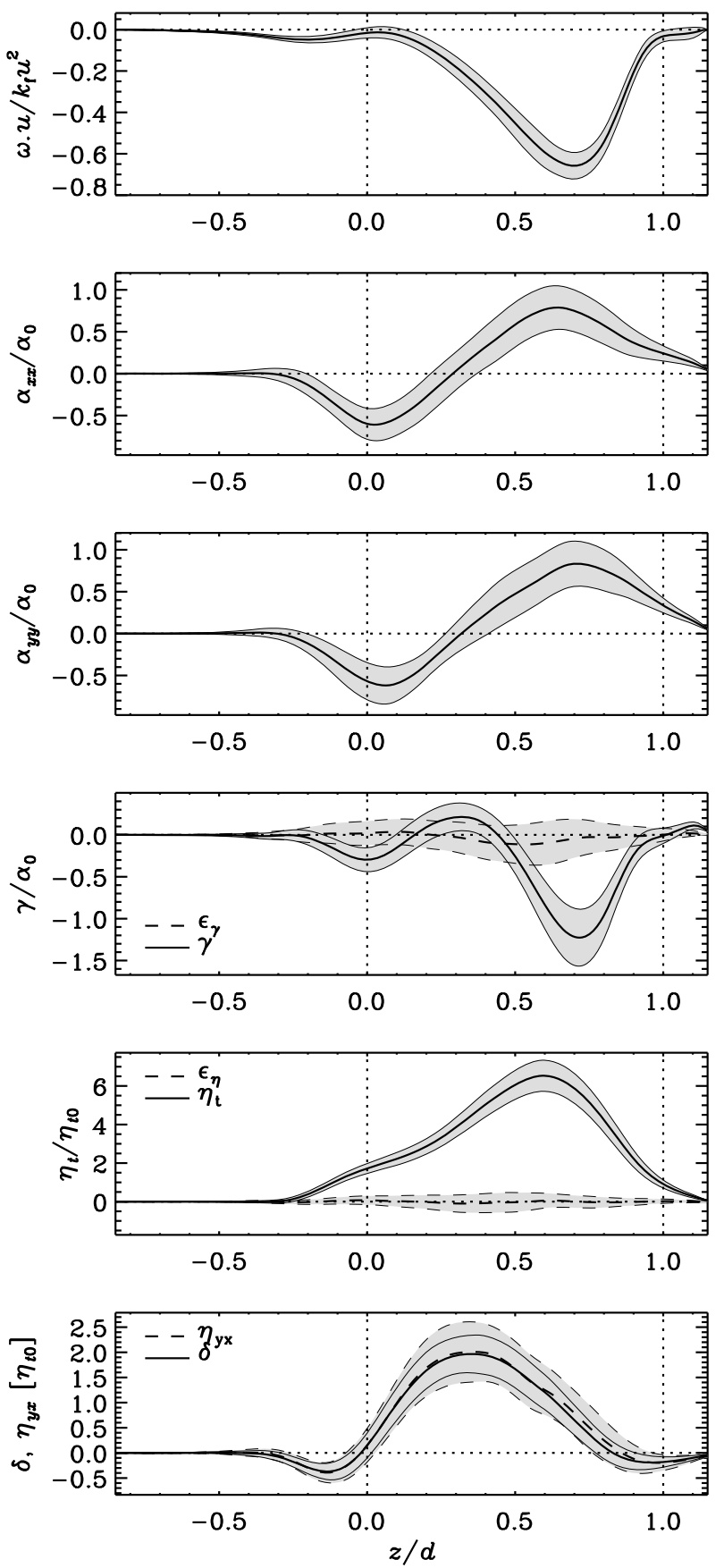

Fig. 3. Same as Fig. 1, but for Run $\mathrm{B}$; $\mathrm{Co} \approx 0.36, \theta=\mathrm{Sh}=0$, and $\mathrm{Rm} \approx 35$.

shown in Fig. 3. Although the number of convection cells in the domain is quite small (of the order of ten or less), the isotropy of $\alpha$ is a preliminary indication that the horizontal system is still large enough to give representative results relevant for a larger ensemble. If the system size is too small, the derived turbulent transport coefficients may no longer be meaningful (Hughes \& Cattaneo 2008). In order to study the convergence of our results, we have performed simulations with three box sizes where the horizontal extent $L_{\mathrm{H}} \equiv L_{x}=L_{y}$ is either $2 d$, $4 d$, or $8 d$, respectively, and the vertical extent of the box is kept unchanged. These runs are labeled (from the smallest to the largest) as B1, B2, and B3, where B2 is the same as Run B (cf. Fig. 3). All three runs are relatively slowly rotating with $\mathrm{Co} \approx 0.36, \mathrm{Sh}=0$, and $\mathrm{Rm} \approx 35$. The results are shown in Fig. 4 . It is obvious that the 

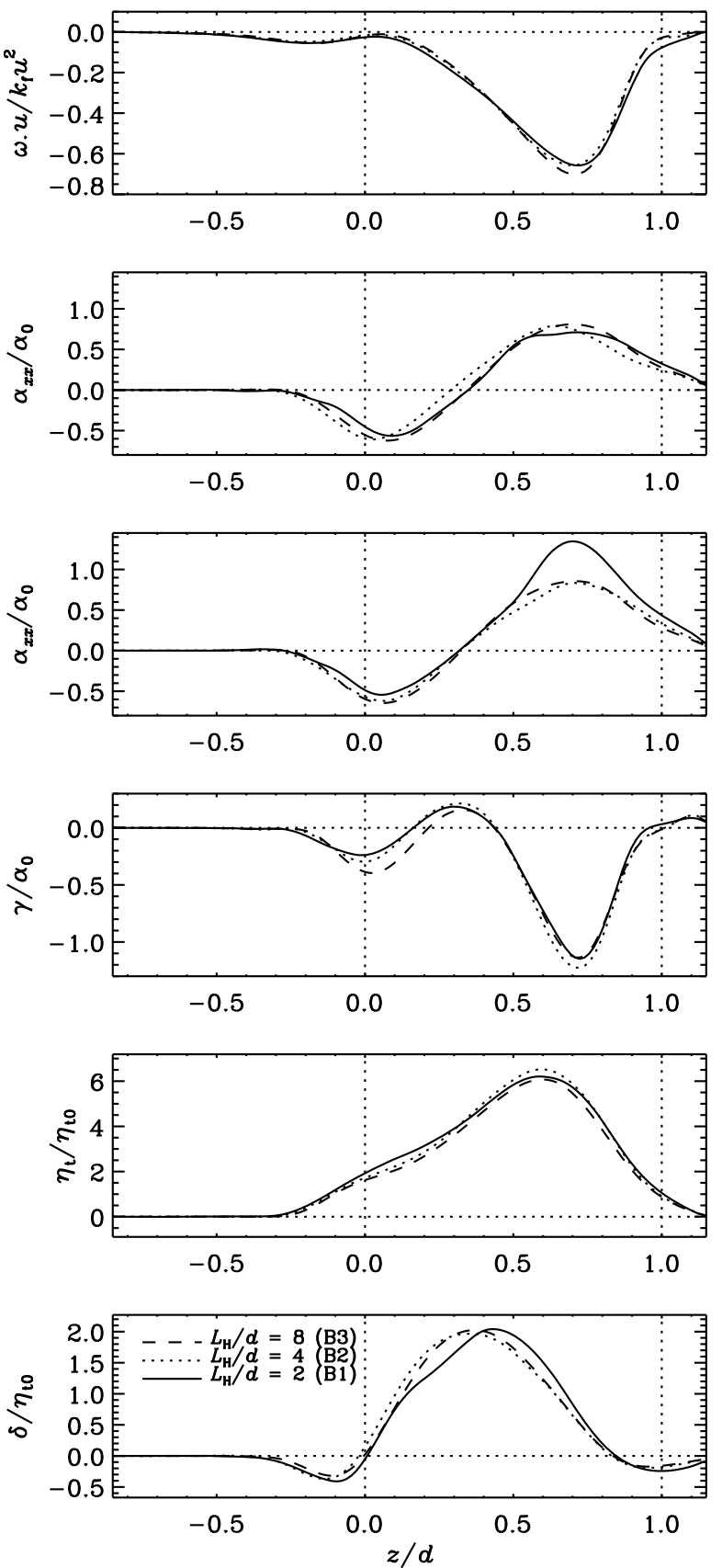

Fig. 4. From top to bottom: kinetic helicity, $\alpha_{x x}, \alpha_{y y}, \gamma, \eta_{\mathrm{t}}$, and $\delta$ as functions of horizontal system size from Runs B1-B3. The linestyles are as indicated in the lowermost panel. $\mathrm{Co} \approx 0.36, \theta=0, \mathrm{Sh}=0$ and $\mathrm{Rm} \approx 35$ in all runs.

differences between the runs are very small and the two larger systems are virtually identical. The only statistically significant difference is the anisotropy of $\alpha$ in the convectively unstable region for Run B1 with the smallest system size. We can thus be fairly confident that the standard box size with $L_{\mathrm{H}}=4 d$ is sufficiently large. This is consistent with the results of Hughes \& Cattaneo (2008) who found that the ratio $L_{\mathrm{H}} / d$ needs to be larger than two for the value of $\alpha$ to be reasonably representative of the system.

\subsubsection{Dependence on $\mathrm{Rm}$}

One of the basic expected properties of turbulent dynamos is that they should be "fast", i.e. the growth rate of the dynamo, and
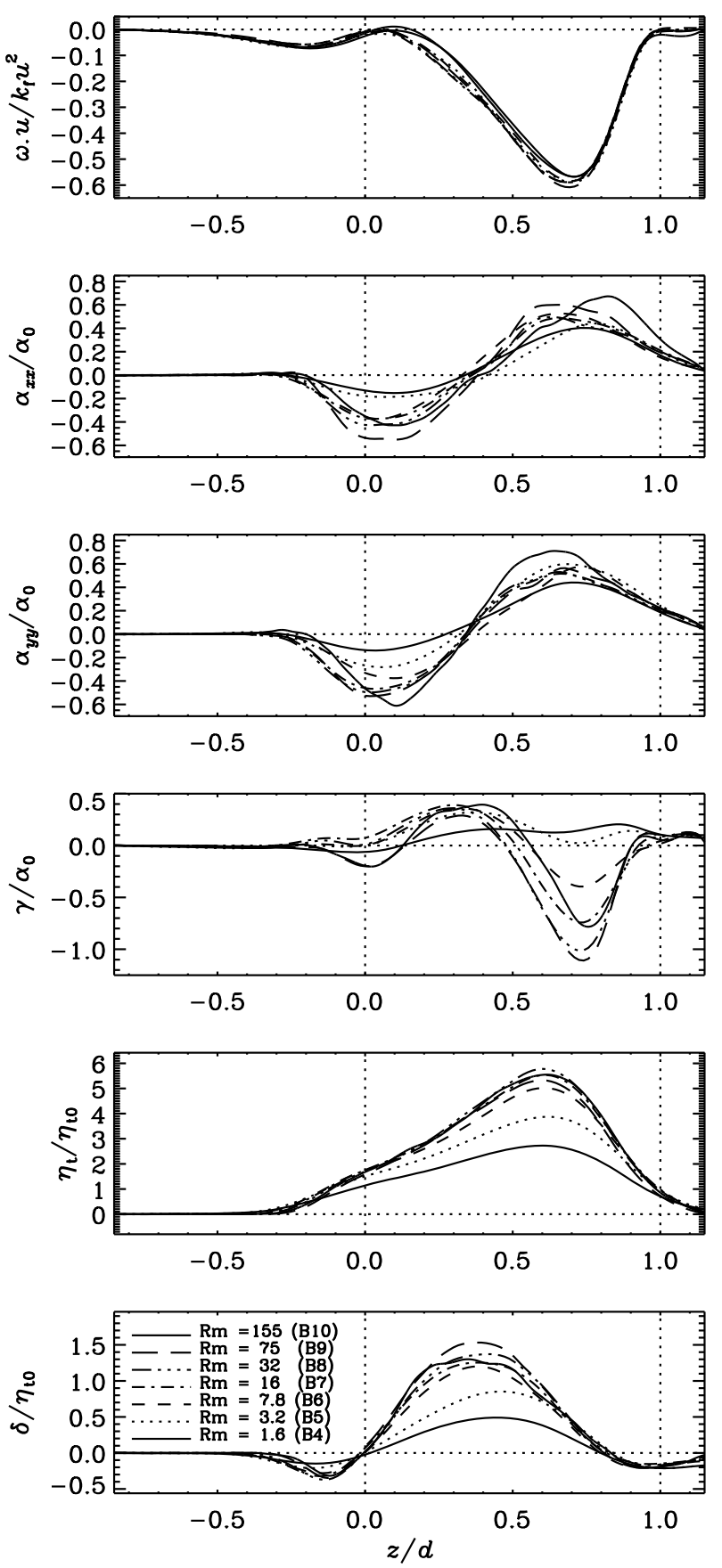

Fig. 5. From top to bottom: kinetic helicity, $\alpha_{x x}, \alpha_{y y}, \gamma, \eta_{\mathrm{t}}$, and $\delta$ as functions of Rm from Runs B4-B10. The linestyles are as indicated in the lowermost panel. $\mathrm{Co} \approx 0.33, \theta=\mathrm{Sh}=0$ and $\mathrm{Re} \approx 15$ in all runs.

thus the transport coefficients, should not depend on the molecular magnetic diffusion provided that $\mathrm{Rm} \gg 1$. Figure 5 shows the transport coefficients as functions of $\mathrm{Rm}$ for fixed $\mathrm{Re} \approx 15$, $\mathrm{Co} \approx 0.33$, and $\theta=\mathrm{Sh}=0$ from Runs B4-B10. The magnetic Reynolds and Prandtl numbers vary in the ranges $1.6 \ldots 155$ and $0.1 \ldots 10$, respectively. We find that the transport coefficients show no statistically significant dependence on $\mathrm{Rm}$ for $\mathrm{Rm} \gtrsim 8$. The only appreciable departures occur for the two lowest Reynolds numbers. However, our definition of the Reynolds number depends on $k_{\mathrm{f}}(=2 \pi / d)$ which is not as well defined as in, e.g., forced turbulence simulations, so the coefficients may depend on $\eta$ for values somewhat larger than $\mathrm{Rm}=1$ in the present case. These results agree with those obtained for isotropic 

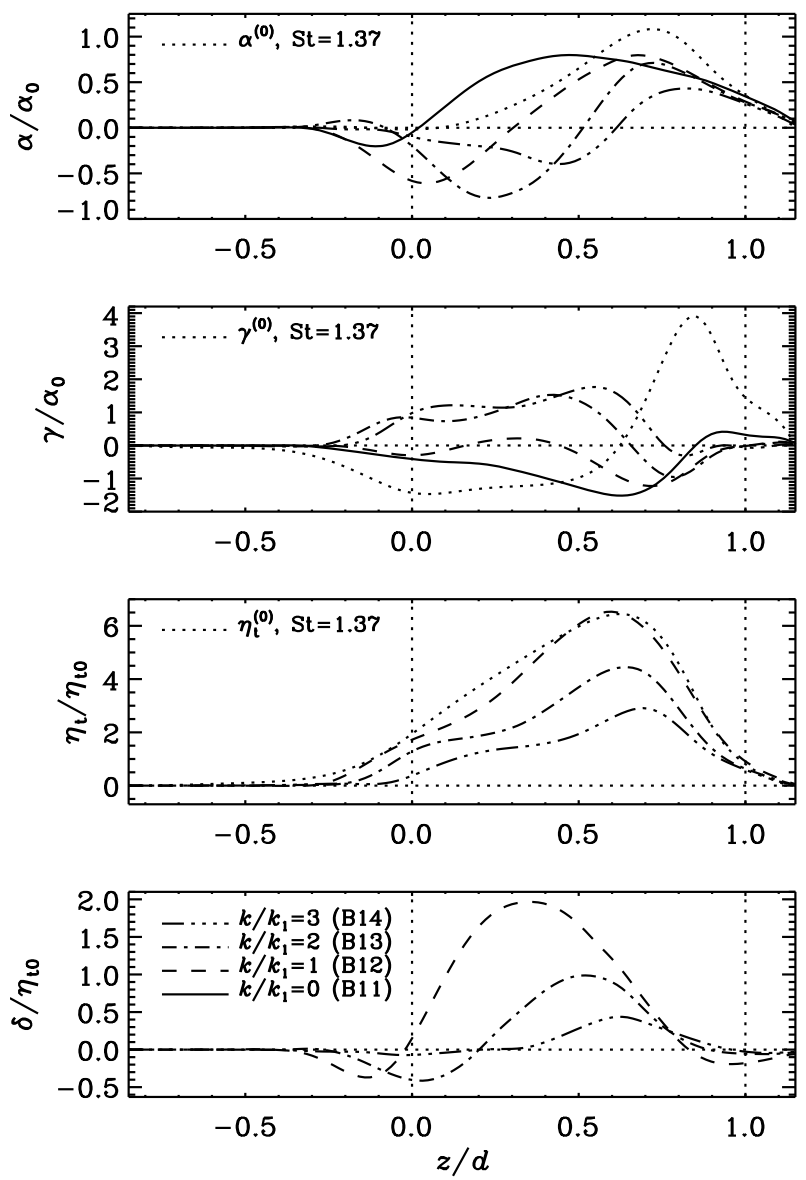

Fig. 6. From top to bottom: $\alpha, \gamma, \eta_{\mathrm{t}}$, and $\delta$ as functions of $k$ for runs $\mathrm{B} 11-\mathrm{B} 14$ with $\mathrm{Rm} \approx 35$ and $\mathrm{Co} \approx 0.36$. Linestyles as indicated in the lowermost panel.

turbulence (Sur et al. 2008; Brandenburg et al. 2008a; Mitra et al. 2009).

\subsubsection{Dependence on wavenumber $k$}

The results for nonrotating convection (see Fig. 2) indicate that at least the pumping effect can experience not only a change in magnitude but also a qualitative change when the wavenumber of the test field is varied (for corresponding details see Brandenburg et al. 2008b). It is of great interest to study whether similar effects can occur for the $\alpha$-effect. Our results for the standard case of $\mathrm{Rm} \approx 35$ and $\mathrm{Co} \approx 0.36$ from runs $\mathrm{B} 11-\mathrm{B} 14$ are shown in Fig. 2. According to symmetry arguments, the diagonal components of $\alpha_{i j}$ are the same for $\theta=0$. We confirm this numerically (see the two previous sections) and combine the data of the $\alpha$-effect into a single coefficient $\alpha$. The same applies to the FOSA expressions, Eqs. (26), (27), which we combine into $\alpha^{(0)}=\frac{1}{2}\left(\alpha_{x x}^{(0)}+\alpha_{y y}^{(0)}\right)$. For $k / k_{1}=0$, the $\alpha$-effect shows a more uniform positive value in the convectively unstable region than for $k / k_{1}=1$. For larger $k$ the negative region in the deeper layers shifts towards the top and the maxima of the profile diminish. The pumping coefficient and turbulent diffusion behave very much the same as in the nonrotating case, cf. Fig. 2. The coefficient $\delta$ diminishes rapidly as $k$ increases. The FOSA expression for the $\alpha$-effect is in qualitative accordance with the $k / k_{1}=0$ result, but fails to capture the details of the profile (see also Käpylä et al. 2006a). The Strouhal number required to match $\eta_{\mathrm{t}}^{(0)}$ with $\eta_{\mathrm{t}}$ is 1.37 which is somewhat smaller than in the nonrotating case.
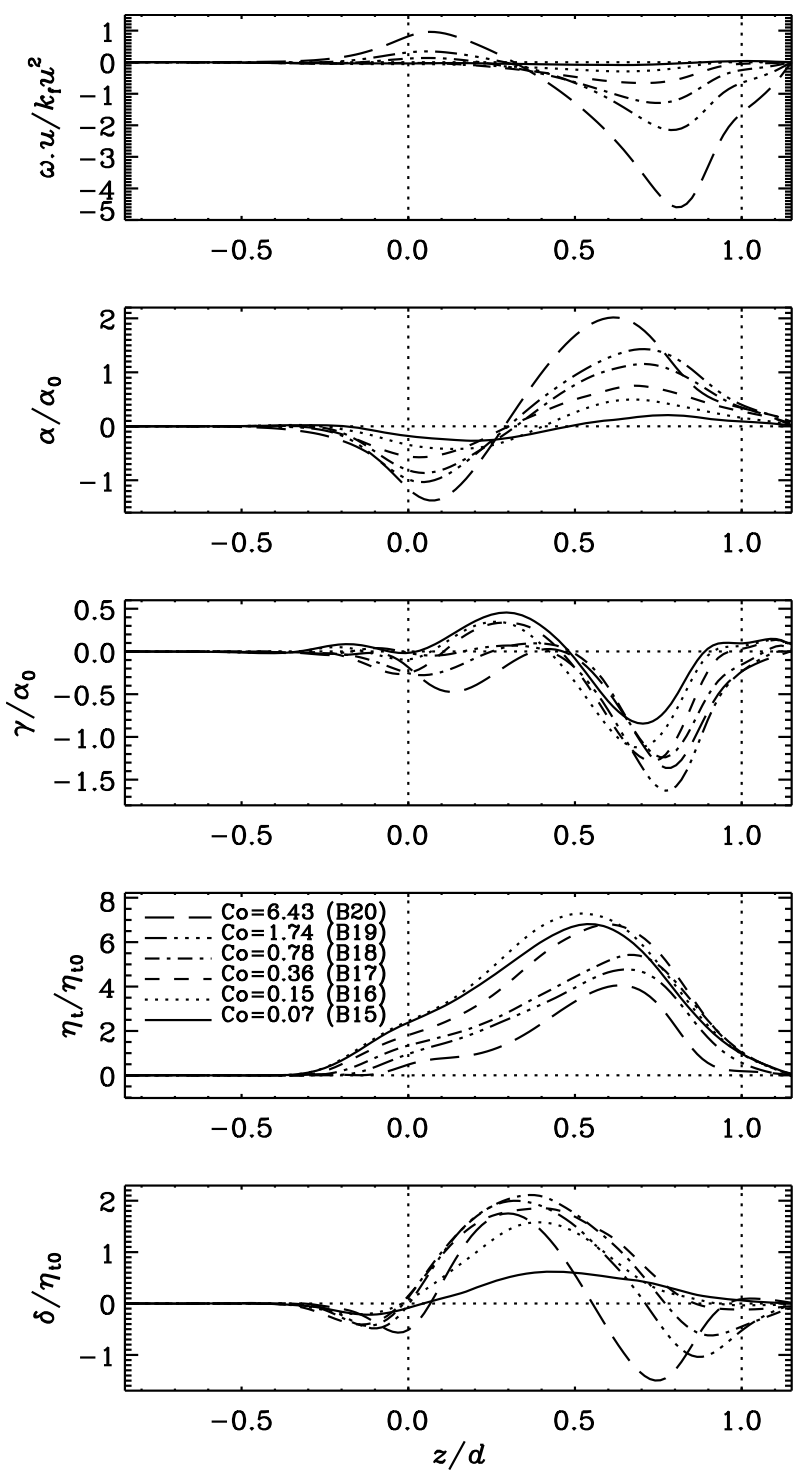

Fig. 7. From top to bottom: kinetic helicity, $\alpha, \gamma, \eta_{\mathrm{t}}$, and $\delta$ as functions of rotation from Runs B15-B20. The linestyles are as indicated in the second panel from the bottom. $\theta=0, \mathrm{Sh}=0$, and $\mathrm{Rm} \approx 20 \ldots 35$.

\subsubsection{Dependence on Co}

The $\alpha$-effect from rotating convection simulations in setups similar to ours has been studied in numerous papers in the past using the imposed field method (e.g. Ossendrijver et al. 2001, 2002; Käpylä et al. 2006a). Our results for the Coriolis number dependence of the kinetic helicity, $\alpha, \gamma, \eta_{\mathrm{t}}$ and $\delta$ from Runs B15-B20 are shown in Fig. 7. We find that the kinetic helicity and $\alpha$ increase monotonically as functions of rotation in accordance with the results of Ossendrijver et al. (2001). The vertical pumping effect shows little dependence on rotation although the Coriolis number changes by two orders of magnitude.

Interestingly, the turbulent diffusivity shows a marked decrease for rapid rotation. The coefficient $\delta$ is positive in the convection zone and negative in the overshoot layer for slow rotation. The magnitude increases rapidly until $\mathrm{Co} \approx 0.2$, after which $\delta$ changes sign near the top. This negative region increases with rotation. Similar results, i.e. monotonically decreasing $\eta_{\mathrm{t}}$ and a first increasing and then decreasing $\delta$ were obtained from forced turbulence simulations by Brandenburg et al. (2008a). 
The combined effect of increasing $\alpha$ and decreasing $\eta_{\mathrm{t}}$ suggests that the large-scale dynamo was possibly subcritical in the runs with only rotation in Paper I and other earlier studies (e.g. Nordlund et al. 1992; Brandenburg et al. 1996; Cattaneo \& Hughes 2006; Tobias et al. 2008), but that it could be excited for more rapid rotation. The validity of this conjecture is given some credibility by Käpylä et al. (2009a) who find clear largescale dynamo action for Co $>4$ for a similar setup as used here and in Paper I. More detailed discussion of these results can be found in the aforementioned reference.

\subsubsection{Dependence on $\theta$}

The latitude dependence of the coefficients for $\mathrm{Co} \approx 0.29-0.36$ and $\mathrm{Rm} \approx 35-43$ from Runs B21-B27 is shown in Fig. 8. The colatitude is varied from $0^{\circ}$ (north pole) to $90^{\circ}$ (equator) in increments of 15 degrees. The kinetic helicity and the diagonal components of $\alpha_{i j}$ decrease monotonically towards the equator. The latter are approximately equal and show a similar latitude dependence. This is consistent with the results of Ossendrijver et al. (2002) and Käpylä et al. (2006a) with a comparable Coriolis number ${ }^{3}$. Vertical pumping also decreases monotonically from the pole to the equator. The equatorial profile of $\gamma$ is quite similar to the nonrotating run, cf. Fig. 1. The trend is similar to that seen in earlier studies, e.g. Käpylä et al. (2006a). The variation of $\eta_{\mathrm{t}}$ is smaller than for the other components, but a weak increasing trend from the pole towards the equator is seen. The coefficient $\delta$ shows a clear decreasing trend as a function of colatitude and is consistent with zero at the equator - in accordance with symmetry considerations.

\subsection{Set $C$ : only shear $(\mathrm{Co}=0, S h \neq 0)$}

\subsubsection{Simulation results}

The next case to consider is that of shear only. We use uniform shear of the form $\bar{U}=(0, S x, 0)$, where $S<0$, resulting in $\boldsymbol{g} \cdot(\boldsymbol{\nabla} \times \overline{\boldsymbol{U}})>0$, and positive kinetic helicity, as expected. See Fig. 9 for representative results from Run $\mathrm{C}$ with $\mathrm{Sh} \approx-0.14$ and $\mathrm{Rm} \approx 46$. We find that introducing shear into the system produces an anisotropic $\alpha$-effect. The profile of the $\alpha_{x x}$ component is similar, but of opposite sign, to that in the case with only rotation; see, e.g., Fig. 3. The magnitude of this component is also quite large, i.e. up to twice the estimate $\alpha_{0}$ already for rather weak shear of $\mathrm{Sh} \approx-0.14$. The $\alpha_{y y}$ coefficient, relevant for dynamo excitation, is positive, but the magnitude is only about one fifth of $\alpha_{x x}$. Moreover, the error bars are so large that the value is hardly statistically significant. These results demonstrate that linking the $\alpha$-effect to the negative of the kinetic helicity can be misleading. Figure 10 shows the coefficients as functions of shear for Runs C1-C4. We have to restrict the study to rather modest values of Sh because shear, in the absence of rotation, promotes generation of large-scale vorticity (e.g. Elperin et al. 2003; Käpylä et al. 2009b). Although our largest value of Sh is still rather modest, the $\alpha_{x x}$ component is quite large, up to three times $\alpha_{0}$. The $\alpha_{y y}$ component, however, remains small and positive for all values of Sh without a consistent trend as a function of shear.

The turbulent pumping in Run $\mathrm{C}$ has a similar profile as in the cases with $\mathrm{Co}=\mathrm{Sh}=0$ (Run A) and $\mathrm{Co} \neq 0$ (Run B) with $k / k_{1}=1$ with downward pumping near the surface and

3 The definition of Coriolis number in the present study is smaller by a factor of $2 \pi$ in comparison to previous studies.
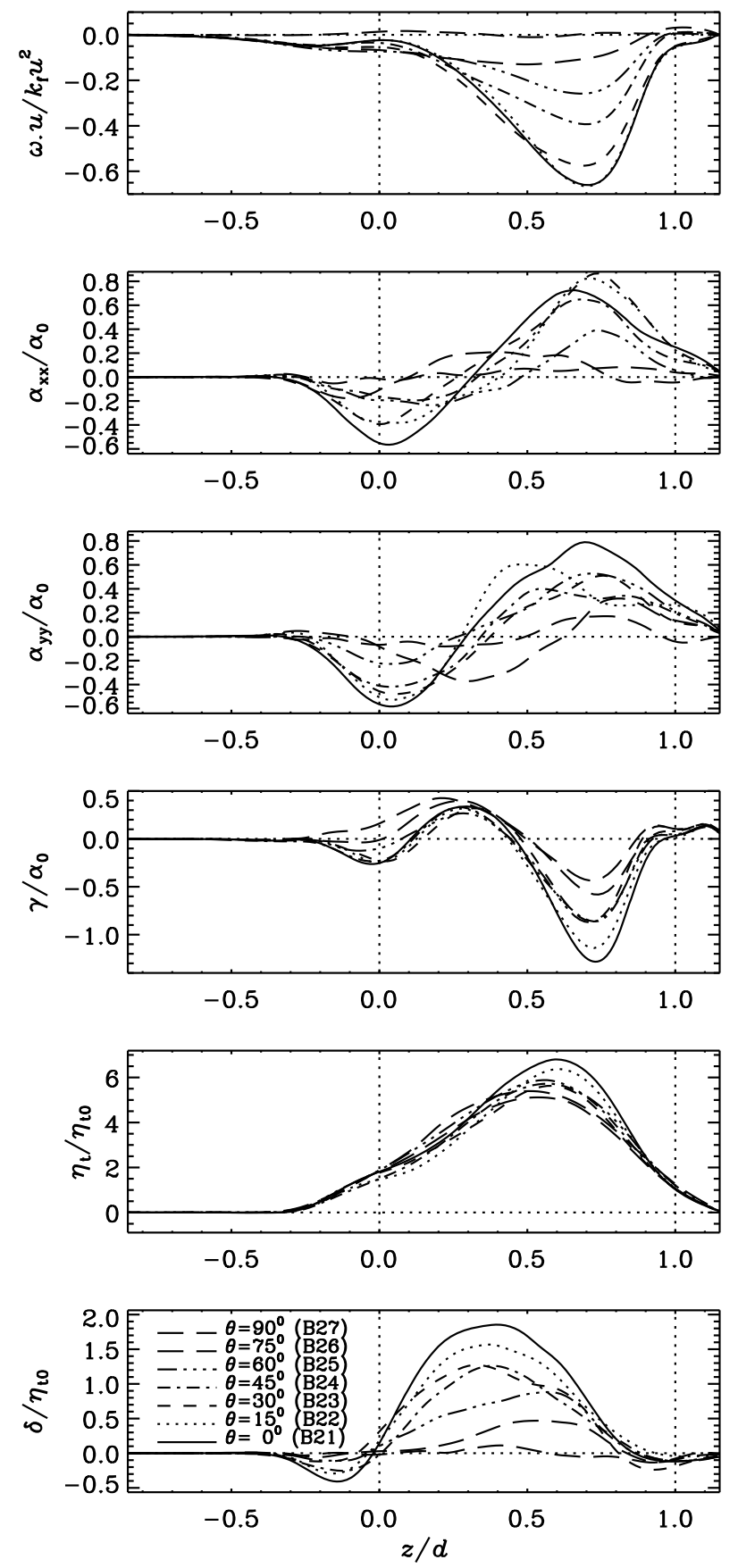

Fig. 8. From top to bottom: kinetic helicity, $\alpha_{x x}, \alpha_{y y}, \gamma, \eta_{\mathrm{t}}$, and $\delta$ as functions of colatitude $\theta$ from Runs B21-B27. The linestyles are as indicated in the lowermost panel. $\mathrm{Co} \approx 0.29-0.36$ and $\mathrm{Rm} \approx 35-43$ in all runs.

upward pumping in the lower part of the convectively unstable region. The profile and magnitude of the turbulent diffusivity is also very similar to previous cases. The pumping effect and turbulent diffusivity are decreased when the magnitude of Sh is greater than 0.06 . The results for increasing $\alpha$ and decreasing $\eta_{\mathrm{t}}$ as functions of shear are opposite to those obtained from helically forced turbulence with shear (Mitra et al. 2009). However, the comparison for the $\alpha$-effect should be done with caution because in Mitra et al. (2009) $\alpha$ arises essentially due to the external forcing and is only modified by the action of shear whereas in the present case $\alpha$ is due to the interaction of shear, stratification, and turbulence themselves. 

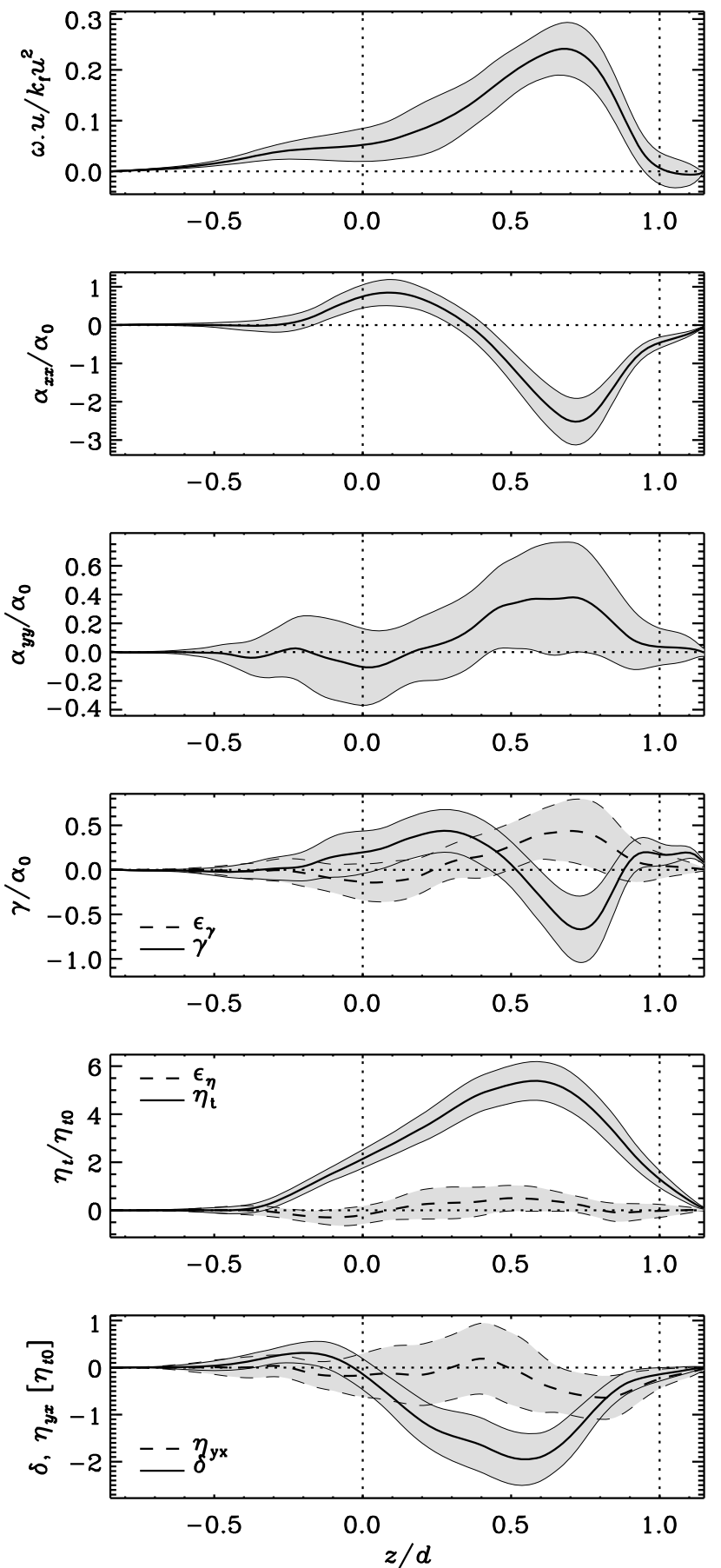

Fig. 9. Same as Fig. 1, but for Run $\mathrm{C}$ with no rotation and just shear; $\mathrm{Co}=0, \mathrm{Sh} \approx-0.14$, and $\mathrm{Rm} \approx 46$

The $\eta_{y x}$ component, which can drive a mean-field shearcurrent dynamo for $\eta_{y x} S>0$, is of interest because it can provide an explanation for the dynamos seen in recent dynamo simulations (Paper I; Hughes \& Proctor 2009). In the present case where $S<0, \eta_{y x}$ should be negative to excite the shear-current dynamo. There appear to be consistently negative regions of $\eta_{y x}$ at the interface of the convectively unstable region and the overshoot layer, and in the upper layers of the convection zone. The upper negative region is more pronounced for $\mathrm{Sh}=-0.06$ and $\mathrm{Sh}=-0.14$. However, the errors of these quantities are of the same order of magnitude as the mean value, cf. the bottom panel of Fig. 9. These results tend to agree with earlier findings from
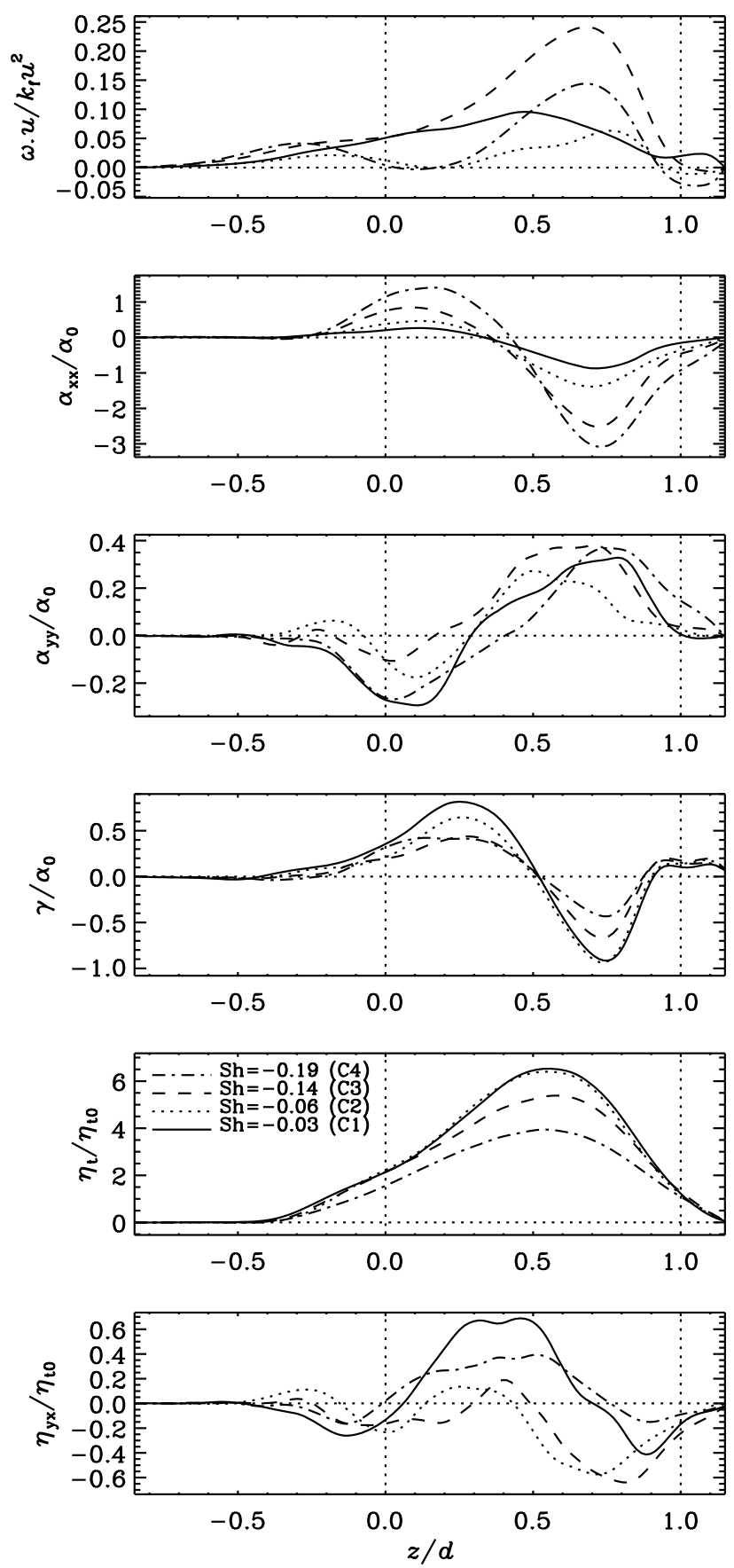

Fig. 10. From top to bottom: kinetic helicity, $\alpha_{x x}, \alpha_{y y}, \gamma, \eta_{\mathrm{t}}$, and $\eta_{y x}$ as functions of shear from Runs $\mathrm{C} 1-\mathrm{C} 4$. The linestyles are as indicated in the second panel from the below. $\mathrm{Co}=0$ and $\mathrm{Rm} \approx 42-46$ in all runs.

forced turbulence (Brandenburg et al. 2008a; Mitra et al. 2009) where $\eta_{y x}$ for the most part was positive or compatible with zero.

\subsubsection{Mean-field dynamo models}

In Paper I clear large-scale dynamo action was found from a simulation with $\mathrm{Sh} \approx-0.08$ whereas for $\mathrm{Sh} \approx-0.03$ the solution was marginal. In the range $-0.08>\mathrm{Sh}>-0.22$ the growth rate of the large-scale field was proportional to the modulus of the shear parameter $S$. Bearing these results in mind and having obtained the turbulent transport coefficients for the 


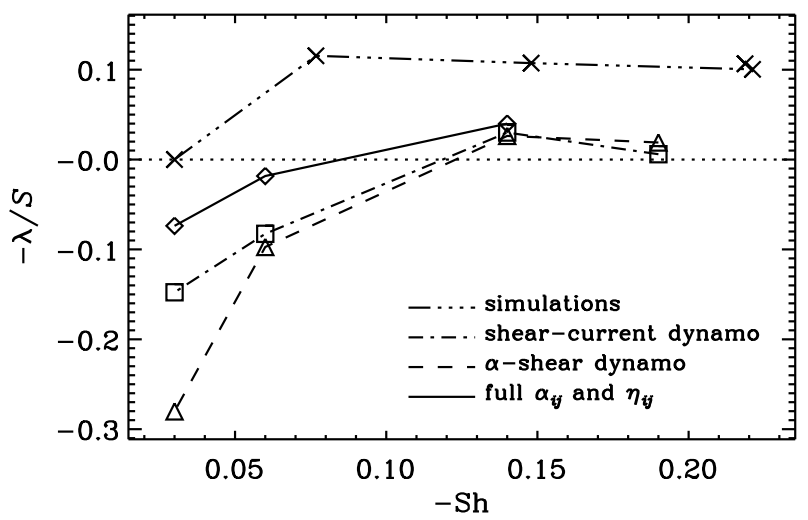

Fig. 11. Growth rates $\lambda$ from a one-dimensional mean-field model with transport coefficients from Runs C1-C4 presented in Fig. 10. Linestyles as indicated in the lower panel.

corresponding parameter regime we are in a position to apply the test field results in a mean-field dynamo model.

Using the full test field results for $\alpha_{i j}$ and $\eta_{i j}$ in the corresponding mean-field model indicates that a dynamo is excited for $-\mathrm{Sh}>0.14$, see the growth rates of the large-scale field presented Fig. 11. It is interesting to study what are the relative importances of the different effects: first we turn off the off-diagonal components of $\eta_{i j}$ in which case the magnetic field is generated by the $\alpha$-effect and the shear-current effect is absent. We find that the growth rate decreases but is still positive for the same cases as before. On the other hand, a "pure" shear-current dynamo, i.e. where $\alpha_{i j}=\eta_{x y}=0$, is also excited for the same runs with a very similar $\lambda$ as in the $\alpha$-shear case. In comparison to the simulations of Paper I, we find that the growth rates from the mean-field model are consistently significantly smaller. These results and the fact that no dynamo was found for $-\mathrm{Sh}=0.06$ would seem to indicate that an incoherent $\alpha$-shear dynamo (e.g. Vishniac \& Brandenburg 1997) is also operating in the full simulations. However, we should remain cautious when comparing the direct simulations and the mean-field model because the transport coefficients were determined for a single value of $k$ whereas many other wavenumbers are available in the simulations.

\subsection{Set $D$ : rotation and shear $(C o \neq 0, S h \neq 0)$}

\subsubsection{Simulation results}

When rotation is added to the system where a large-scale shear is already imposed, the vorticity generation is suppressed (e.g. Yousef et al. 2008b; Paper I) and it is possible to study higher values of Sh. Representative results for Run $\mathrm{D}$ with $\mathrm{Co} \approx 0.36$, $\mathrm{Sh} \approx-0.18$, and $\mathrm{Rm} \approx 35$ are shown in Fig. 12. The results for the kinetic helicity and $\alpha_{y y}$ seem to behave additively when comparing with the runs with only rotation (Run B; Fig. 3) and only shear (Run C; Fig. 9). The $\alpha_{x x}$ component is somewhat smaller than in Run $\mathrm{C}$ with shear only which is consistent with oppositely signed contributions due to shear and rotation.

We note that in a recent paper, Hughes \& Proctor (2009) found that the $\alpha$-effect is virtually unchanged when shear is added to a rotating system. In their case the shear profile is proportional to $\cos y$. The resulting large-scale vorticity is then $\bar{W}_{z} \propto \sin y$ which leads to $\alpha_{i j}^{(W)} \propto(\boldsymbol{G} \cdot \overline{\boldsymbol{W}}) \delta_{i j} \propto \sin y$ (e.g. Rädler $\&$ Stepanov 2006) where $\boldsymbol{G}$ symbolically denotes the inhomogeneity of the turbulence. However, Hughes \& Proctor (2009) show a volume average of $\alpha$ over the full upper half of the domain in which case the contribution of $\alpha^{(W)}$ cancels out. This
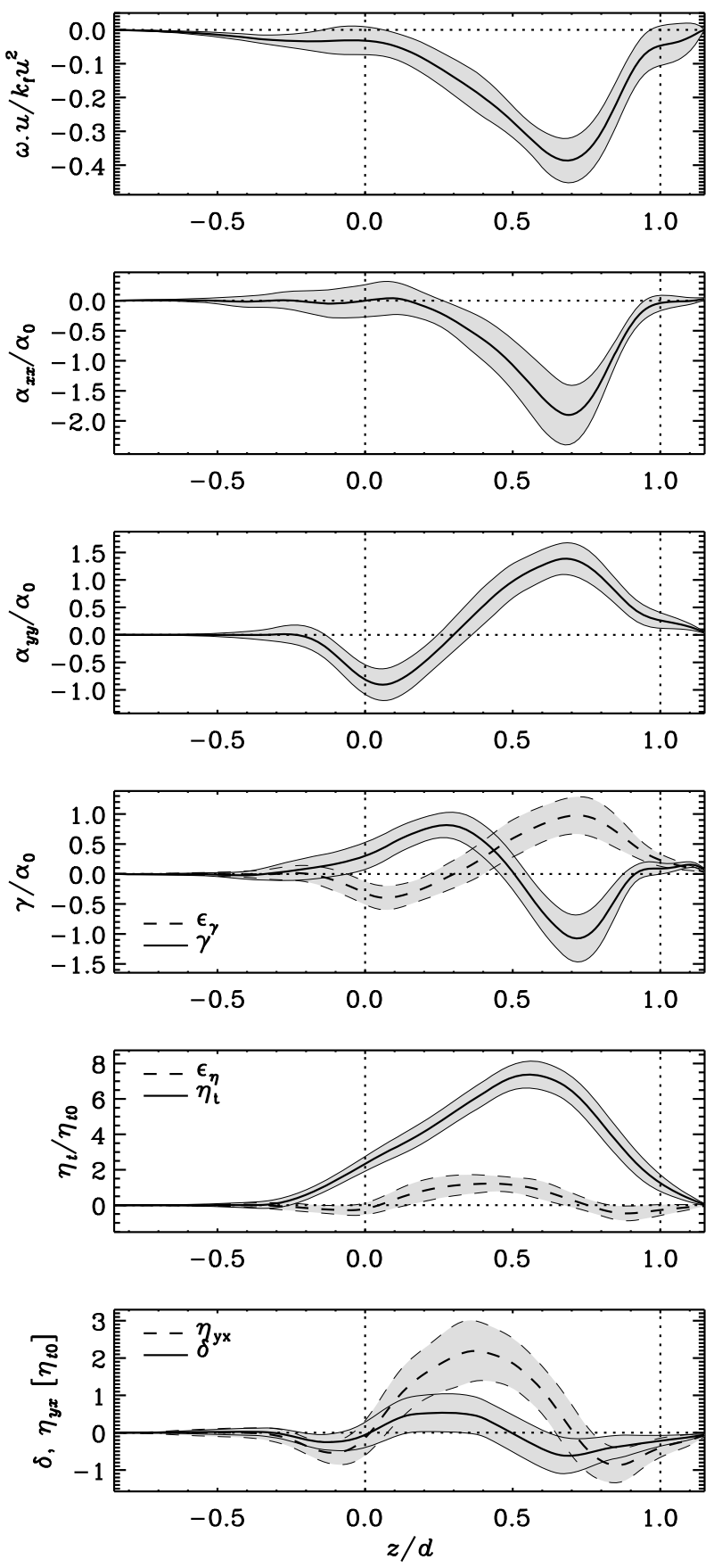

Fig. 12. Same as Fig. 1, but for Run D with both rotation and shear; $\mathrm{Co} \approx 0.36, \mathrm{Sh} \approx-0.18$, and $\mathrm{Rm} \approx 37$.

explains the absence of any modifications of $\alpha$ due to shear in their case, but for us this is not the case because for our shear profile $\bar{W}_{z}=S=$ const.

The profile of $\gamma$ is quite similar to the rotating case, i.e. Run B; see Fig. 3, with the exception that the off-diagonal components of $\alpha_{i j}$ show considerable anisotropy as manifested by the parameter $\epsilon_{\gamma}$. The turbulent diffusion shows a profile common to all the other simulations, but here the diagonal components of $\eta_{i j}$ show evidence of mild anisotropy with $\epsilon_{\eta}$ peaking near the middle of the convectively unstable region with a maximum value of $\epsilon_{\eta} \approx \eta_{\mathrm{t} 0}$. The quantity $\delta$ is compatible with zero whereas $\eta_{y x}$ exhibits a similar profile and magnitude as $\delta$ does in the rotating case, cf. Fig. 3, which indicates that $\eta_{x y} \approx \eta_{y x}$, as opposed to $\eta_{x y} \approx-\eta_{y x}$ in Run B. 

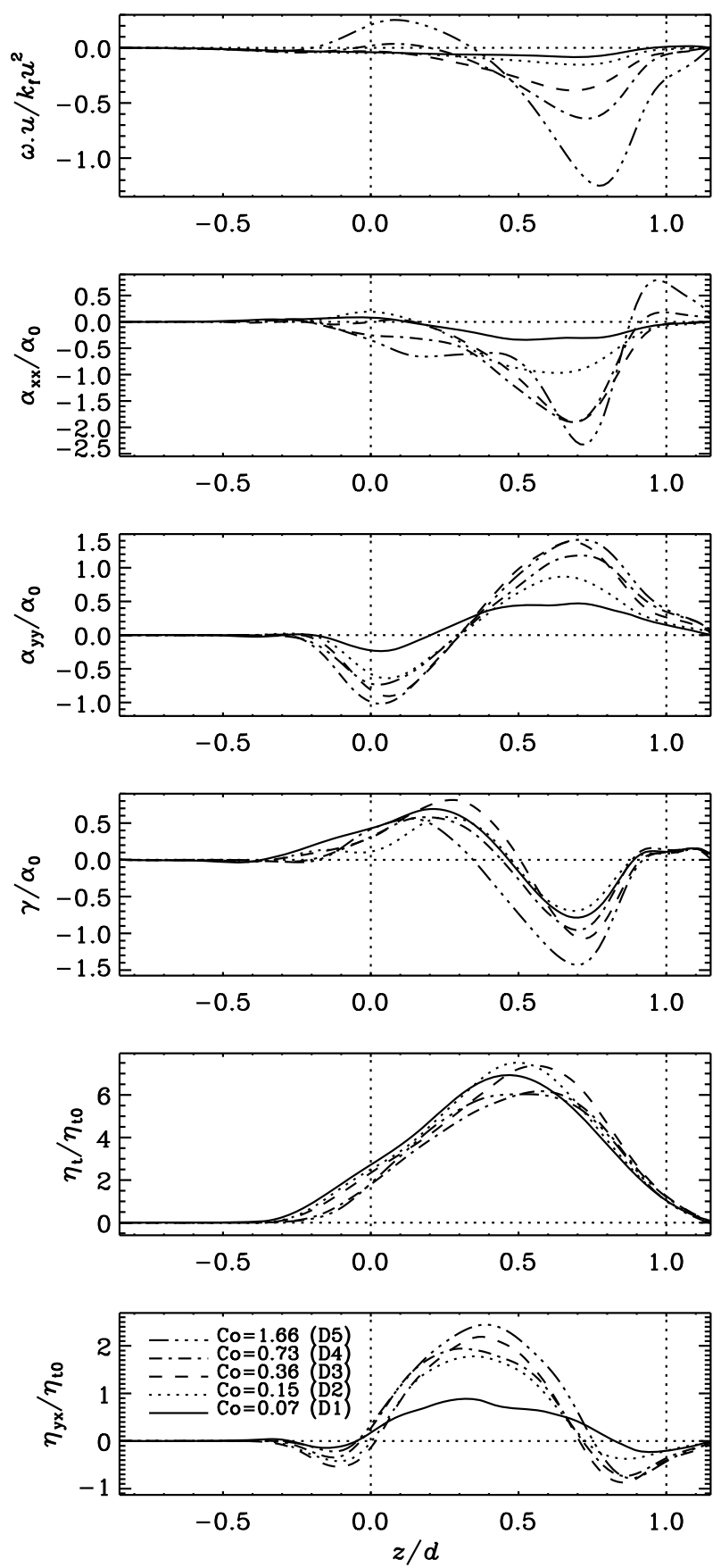

Fig. 13. From top to bottom: kinetic helicity, $\alpha_{x x}, \alpha_{y y}, \gamma, \eta_{\mathrm{t}}$, and $\eta_{y x}$ as functions of Coriolis number for Runs D1-D5. The linestyles are as indicated in the lowermost panel. $\mathrm{Sh}=-\frac{1}{2} \mathrm{Co}$ and $\mathrm{Rm} \approx 37$ in all runs.

The kinetic helicity and the turbulent transport coefficients as functions of Co, keeping the ratio $-S / \Omega=1$ constant, are shown in Fig. 13. The helicity is increasing in a similar fashion as, albeit slower than, in the absence of shear, compare with Fig. 7. The components of the $\alpha$-effect are highly anisotropic with the main contribution of $\alpha_{x x}$ being due to shear and that of $\alpha_{y y}$ due to rotation (compare with Figs. 7 and 10). The value of $\alpha_{x x}$ is somewhat decreased in comparison to the cases with shear only whereas $\alpha_{y y}$ is almost unaffected. This is in qualitative agreement with adding the contributions of runs from Sets $\mathrm{B}$ and $\mathrm{C}$ with corresponding Co and Sh, respectively.

The profiles of $\gamma$ and $\eta_{\mathrm{t}}$ are similar to those with only rotation. The differences of $\gamma$ and $\eta_{\mathrm{t}}$ as a function of Co are small and

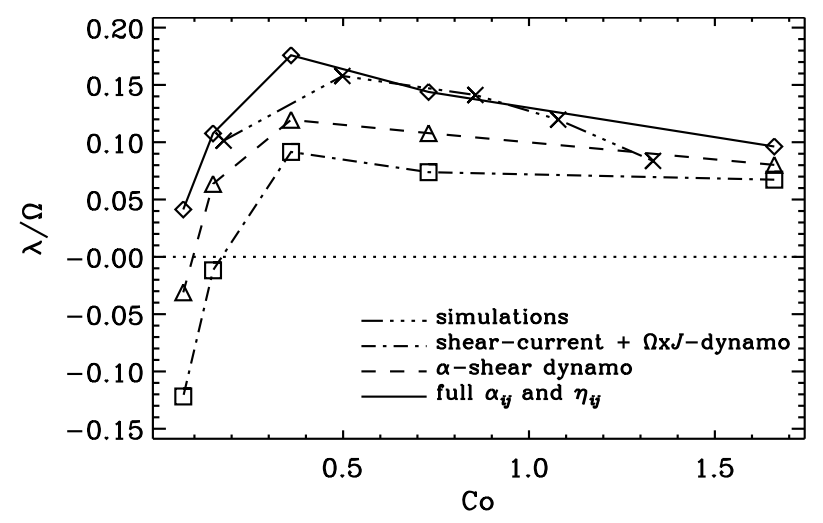

Fig. 14. Same as Fig. 11 but for Runs D1-D5 shown in Fig. 13.

for the most part fall within the error bars. The profile of $\eta_{y x}$ is very similar to $\delta$ in the case of only rotation with negative values near the base and top of the convectively unstable region with positive values in between. The profile and magnitude of $\eta_{y x}$ remains essentially fixed for $\mathrm{Co} \gtrsim 0.15$. We note that in the simulations with shear and rotation the $\boldsymbol{\Omega} \times \boldsymbol{J}$-effect may also contribute to the generation of large-scale magnetic fields (e.g. Rädler 1969; Rädler et al. 2003; Pipin et al. 2008). It is, however, not altogether clear how to disentangle the transport coefficients responsible for the shear-current and $\boldsymbol{\Omega} \times \boldsymbol{J}$-dynamos in the present case. Thus the off-diagonal components of $\eta_{i j}$ contain contributions from both effects in Set D.

\subsubsection{Mean-field dynamo models}

We follow here the same procedure as in Sect. 3.3.2 to study dynamo excitation for the Runs D1-D5. Using the full $\alpha_{i j}$ and $\eta_{i j}$ tensors in the one-dimensional mean-field model indicates that all of the runs in Fig. 13 are capable of driving a dynamo. Neglecting the off-diagonal components of $\eta_{i j}$ decreases $\lambda$ by approximately a third whereas for a pure shear-current dynamo the growth rate is roughly half of the model where the full $\alpha_{i j}$ and $\eta_{i j}$ tensors were used. For an $\alpha$-shear dynamo $\lambda$ is positive for all runs except the slowest rotation case with $\mathrm{Co}=0.07$ whereas for the combined shear-current and $\boldsymbol{\Omega} \times \boldsymbol{J}$-dynamo also Co $=0.15$ is mildly subcritical. It is interesting to note that the simulation results of Paper I fall roughly on top of the uppermost line, i.e. where the full $\alpha_{i j}$ and $\eta_{i j}$ are used, in Fig. 14. This is in contrast to the case of only shear where the growth rates from the mean-field model were clearly smaller than in the corresponding simulations of Paper I. However, the mean-field model does not reproduce the declining growth rate for $\mathrm{Co} \gtrsim 1.1$ that is observed in the direct simulations.

\section{Conclusions}

We obtain turbulent transport coefficients governing the evolution of large-scale magnetic fields from turbulent convection simulations with the test field method. We study the system size and magnetic Reynolds number dependences of the coefficients. This is important because spurious results can be expected for small Reynolds numbers or when the aspect ratio of the domain is too small (Hughes \& Cattaneo 2008). We find that for our standard system size, $L_{\mathrm{H}} / d=4$, the coefficients are essentially identical to those obtained with a horizontal extent that is twice as large. As a function of $\mathrm{Rm}$, all the coefficients are essentially constant for $\mathrm{Rm} \gtrsim 8$. This is in accordance with the theory but 
at odds with results from certain imposed field calculations (e.g. Cattaneo \& Hughes 2006). In these calculations the magnetic field is allowed to evolve until saturation which can cause strong quenching even if the imposed field itself is weak. This is particularly important if closed boundary conditions for the magnetic field are imposed, in which case magnetic helicity conservation can lead to catastrophic quenching (Vainshtein \& Cattaneo 1992). More reliable results for the kinematic $\alpha$-effect with the imposed field method can be obtained by resetting the magnetic field before it grows too large or develops substantial gradients (e.g. Ossendrijver at al. 2002; Käpylä et al. 2006a). More detailed comparison of the imposed field and test field methods is important, but beyond the scope of the present study.

The earlier determinations of transport coefficients from convection simulations have used the imposed field method (e.g. Ossendrijver et al. 2001, 2002; Käpylä et al. 2006a) which yields the components of $\alpha_{i j}$ but does not deliver $\eta_{i j}$ because the imposed field is uniform. The test field method does not suffer from this restriction and $\eta_{i j}$ and the $k$-dependence of the coefficients can be extracted. We find that for $k / k_{1}=0$, i.e. for a uniform field, the results for $\alpha$ and $\gamma$ are consistent with those obtained from imposed field calculations, provided the magnetic field is reset before it grows too large and substantial gradients develop. As $k$ is increased, however, the qualitative behaviour of the coefficients changes. This is indicated by a partial sign change of $\alpha$ and a complete sign change of $\gamma$; see Figs. 2 and 6.

The turbulent diffusivity shows a robust behaviour regardless of the parameters of the simulations: the profile is proportional to the vertical velocity squared, $\overline{u_{z}^{2}}$, as predicted by FOSA (e.g. Rädler 1980). The value of $\eta_{\mathrm{t}}$ decreases almost proportional to $k^{-1}$, and shows a declining trend as a function of rotation and shear.

For the present parameters, the $\alpha$-effect increases monotonically as rotation is increased. As a function of latitude, the diagonal components of $\alpha_{i j}$ have a similar magnitude and peak near the pole with declining values towards the equator. The $\alpha$-effect induced by shear is highly anisotropic: the $\alpha_{x x}$ component has a similar profile and magnitude, but opposite sign, as $\alpha_{x x}$ and $\alpha_{y y}$ in the case of only rotation. This component also increases monotonically as a function of shear, whereas the shear-induced $\alpha_{y y}$ remains small regardless of the strength of the shear. In the runs where rotation and shear are present, the diagonal components of $\alpha_{i j}$ are roughly the sums of the corresponding coefficients in the cases with rotation and shear alone.

In addition to the $\alpha$-effect, the $\eta_{y x}$ component can contribute to a shear-current dynamo when $\eta_{y x} S>0$. In our case, where $S<0$, such dynamo action is possible if $\eta_{y x}<0$. We find that this coefficient shows negative regions near the base and near the top of the convectively unstable region, but the errors are of the same order of magnitude as the negative mean values in most cases.

In order to connect to earlier work, we use the test field results in a one-dimensional mean-field model in order to understand the excitation of dynamos using identical setups as in direct simulations (Paper I). We study here only the cases with shear and consider large-scale dynamos in the rigidly rotating case elsewhere (Käpylä et al. 2009a). The presently used dynamo model ignores $k$-dependence and is therefore likely to be too simple to fully describe the large-scale fields in the direct simulations. Nevertheless, the present results, taken at face value, seem to indicate that in the case with shear alone the derived dynamo coefficients are not sufficient to explain the dynamo but that an additional incoherent $\alpha$-shear dynamo might be needed. This conjecture is based on the fact that mean-field $\alpha$-shear and shear-current dynamos are both excited with similar growth rates which, however, are significantly smaller than those obtained from direct simulations in Paper I. Furthermore, a large-scale dynamo was marginal for $\mathrm{Sh}=-0.03$ in Paper I, whereas for $\mathrm{Sh}=-0.06$ it was found to be slightly subcritical in the present study. On the other hand, for the case with both shear and rotation, no additional incoherent effects seem to be needed. We find that in this case the regular $\alpha$-shear dynamo produces larger growth rates than the combined shear-current and $\mathbf{\Omega} \times \boldsymbol{J}$ dynamo but neither effect alone seems to be strong enough to explain the dynamos in Paper I.

On a more general level, mean-field dynamo models of the Sun and other stars rely on parameterisations of turbulent transport coefficients. Even today, the majority of solar dynamo models bypass this problem and ignore most of the turbulent effects and rely on phenomenological descriptions of the $\alpha$-effect and turbulent diffusion that are not without problems theoretically. On the other hand, some attempts have been made to incorporate the results for the transport coefficients from imposed field studies in mean-field models of the solar magnetism (e.g. Käpylä et al. 2006b; Guerrero \& de Gouveia Dal Pino 2008) and models employing more general turbulence models have recently appeared (e.g. Pipin \& Seehafer 2009). We feel that this is a worthy cause to follow further with the present results.

Acknowledgements. The authors wish to acknowledge the anonymous referee and Prof. Gunther Rüdiger for their helpful comments on the manuscript. The computations were performed on the facilities hosted by CSC - IT Center for Science in Espoo, Finland, who are administered by the Finnish ministry of education. This research has greatly benefitted from the computational resources granted by the CSC to the grand challenge project "Dynamo08". Financial support from the Academy of Finland grants No. 121431 (PJK) and 112020 (MJK) and the Swedish Research Council grant 621-2007-4064 (AB) is acknowledged, The authors acknowledge the hospitality of Nordita during the program "Turbulence and Dynamos" during which this work was initiated.

\section{References}

Brandenburg, A. 2001, ApJ, 550, 824

Brandenburg, A. 2005a, ApJ, 625, 539

Brandenburg, A. 2005b, AN, 326, 787

Brandenburg, A. 2008, AN, 329, 725

Brandenburg, A., \& Käpylä, P. J. 2007, NJP, 9, 305

Brandenburg, A., \& Subramanian, K. 2005, Phys. Rep., 417, 1

Brandenburg, A., Tuominen, I., Nordlund, A., et al. 1990, A\&A, 232, 277

Brandenburg, A., Nordlund, A., Stein, R. F., \& Torkelsson, U. 1995, ApJ, 446, 741

Brandenburg, A., Jennings, R. L., Nordlund, Å., et al. 1996, JFM, 306, 325 Brandenburg, A., Bigazzi, A., \& Subramanian, K. 2001, MNRAS, 325, 685 Brandenburg, A., Rädler, K.-H., Rheinhardt, M., \& Käpylä, P. J. 2008a, ApJ, 676,740

Brandenburg, A., Rädler, K.-H., \& Schrinner, M. 2008b, A\&A, 482, 739

Brown, B. P., Browning, M. K., Brun, A. S., et al. 2007, AIPC, 948, 271

Browning, M. K., Miesch, M. S., Brun, A. S., \& Toomre, J. 2006, ApJ, 648, L157

Cattaneo, F., \& Hughes, D. W. 2006, JFM, 553, 401

Elperin, T., Kleeorin, N., \& Rogachevskii, I. 2003, PhRvE, 68, 016311

Giesecke, A., Ziegler, U., \& Rüdiger, G. 2005, Phys. Earth Planet. Interiors, 152, 90

Gressel, O., Ziegler, U., Elstner, D., \& Rüdiger, G. 2008, AN, 329, 619401

Guerrero, G., \& de Gouveia Dal Pino, E. M. 2008, A\&A, 485, 267

Hughes, D. W., \& Cattaneo, F. 2008, JFM, 594, 445

Hughes, D. W., \& Proctor, M. R. E. 2009, PhRvL, 102, 044501

Hawley, J. F., Gammie, C. F., \& Balbus, S.A. 1996, ApJ, 464, 690

Käpylä, P. J., \& Brandenburg, A. 2009, ApJ, in press [arXiv: 0810.2298]

Käpylä, P. J., Korpi, M. J., \& Tuominen, I. 2004, A\&A, 422, 793

Käpylä, P. J., Korpi, M. J., Ossendrijver, M., \& Stix, M. 2006a, A\&A, 455, 401

Käpylä, P. J., Korpi, M. J., \& Tuominen, I. 2006b, AN, 327, 884

Käpylä, P. J., Korpi, M. J., \& Brandenburg, A. 2008, A\&A, 491, 353 (Paper I)

Käpylä, P. J., Korpi, M. J., \& Brandenburg, A. 2009a, ApJ, 697, 1153 
Käpylä, P. J., Mitra, D., \& Brandenburg, A. 2009b, PhRvE, 79, 016302 Kleeorin, N., \& Rogachevskii, I. 2008, PhRvE, 77, 036307

Krause, F., \& Rädler, K.-H. 1980, Mean-field Magnetohydrodynamics and Dynamo Theory (Oxford: Pergamon Press)

Mininni, P. D., Gómez, D. O., \& Mahajan, S. M. 2005, ApJ, 619, 1019

Mitra, D., Käpylä, P. J., Tavakol, R., \& Brandenburg, A. 2009, A\&A, 495, 1

Moffatt, H. K. 1978, Magnetic field generation in electrically conducting fluids (Cambridge: Cambridge Univ. Press)

Nordlund, Å, Brandenburg, A., Jennings, R. L., et al. 1992, ApJ, 392, 647

Ossendrijver, M. 2003, A\&AR, 11, 287

Ossendrijver, M., Stix, M., \& Brandenburg, A. 2001, A\&A, 376, 726

Ossendrijver, M., Stix, M., Rüdiger, G., \& Brandenburg, A. 2002, A\&A, 394, 735

Parker, E. N. 1979, Cosmical Magnetic Fields: Their Origin and Their Activity (Oxford, NY: Clarendon Press)

Pipin, V. V. 2008, GAFD, 102, 21

Pipin, V. V., \& Seehafer, N. 2009, A\&A, 493, 819

Proctor, M. R. E. 2007, MNRAS, 382, 39

Rädler, K.-H. 1968, Z. Naturforsch., 23a, 1851

Rädler, K.-H. 1969, Monatsber. Dtsch. Akad. Wiss. Berlin, 11, 194

Rädler, K.-H. 1980, AN, 301, 101

Rädler, K.-H., \& Stepanov, R. 2006, PhRvE, 73, 056311
Rädler, K.-H., Kleeorin, N., \& Rogachevskii, I. 2003, GAFD, 97, 249

Rogachevskii, I., \& Kleeorin, N. 2003, PhRvE, 68, 036301

Rogachevskii, I., \& Kleeorin, N. 2004, PhRvE, 70, 046310

Rotvig, J., \& Jones, C. A. 2002, PhRvE, 66, 056308

Rüdiger, G., \& Hollerbach, R. 2004, The magnetic Universe (Weinheim: Wiley$\mathrm{VCH})$

Rüdiger, G., \& Kitchatinov, L. L. 2006, AN, 327, 298

Schrinner, M., Rädler, K.-H., Schmitt, D., et al. 2005, AN, 326, 245

Schrinner, M., Rädler, K.-H., Schmitt, D., et al. 2007, GAFD, 101, 81

Sur, S., Brandenburg, A., \& Subramanian, K. 2008, MNRAS, 385, L15

Tobias, S. M., Brummell, N. H., Clune, Th. L., \& Toomre, J. 1998, ApJ, 502, L177

Tobias, S. M., Brummell, N. H., Clune, Th. L., \& Toomre, J. 2001, ApJ, 549, 1183

Tobias, S. M., Cattaneo, F., \& Brummell, N. H. 2008, ApJ, 685, 596

Vainshtein, S. I., \& Cattaneo, F. 1992, ApJ, 393, 165

Vishniac, E. T., \& Brandenburg, A. 1997, ApJ, 475, 263

Vishniac, E. T., \& Cho, J. 2001, ApJ, 550, 752

Yousef, T. A., Heinemann, T., Schekochihin, A. A., et al. 2008a, PhRvL, 100, 184501

Yousef, T. A., Heinemann, T., Rincon, F., et al. 2008b, AN, 329, 737

Ziegler, U., \& Rüdiger, G. 2003, A\&A, 401, 433 